\title{
ARTICLE \\ The efficacy of positive psychology interventions from non-Western countries: A systematic review and meta- analysis
}

\author{
Tom Hendriks · Marijke Schotanus-Dijkstra · Aabidien Hassankhan · Tobi Graafsma • \\ Ernst Bohlmeijer · Joop de Jong
}

\begin{abstract}
Recently, there has been a sharp increase in the number of studies of positive psychology interventions (PPIs) from non-Western countries. The aim of this study is to review and evaluate the efficacy of these PPIs. Databases, including PubMed, PsycINFO, and Scopus, were searched up to December 2017. In addition, we performed hand searches and reference checks. After removal of duplicates, 7,516 studies were screened and finally 28 randomized controlled trials (RCTs) were included in the meta-analysis. A random effects model was used to compare group effect-sizes at post-test. Results showed that PPIs from non-Western countries have a moderate effect on subjective wellbeing $(g=0.48)$ and psychological wellbeing $(g=0.40)$, and a large effect on depression $(g=0.62)$ and anxiety $(g=0.95)$. However, caution is warranted for the interpretation of the effect sizes in light of the study quality, which was assessed as low. This indicates the possibility of biases, which may explain why PPIs from non-Western countries report larger effect sizes than PPIs from Western countries.
\end{abstract}

Keywords: positive psychology interventions, wellbeing, positive mental health, cross-cultural

\section{Introduction}

In the past decade, there has been a rapidly growing number of studies investigating the effects of positive psychology interventions (PPIs) (Rusk \& Waters, 2013). To date, it appears that consensus on the definition of PPIs has not yet been reached. Sin and Lyubomirsky (2009) introduced a broad definition, defining PPIs as all interventions that are aimed at increasing positive feelings, behaviors, and cognition. Narrower definitions were suggested by Bolier et al. (2013), who added that these interventions should have been explicitly developed in line with the theoretical tradition of positive psychology, and Parks and Biswas-Diener, who suggested that an intervention can only be regarded as a PPI if sufficient empirical evidence exists suggesting significant effects for the intervention (Parks \& Biswas-Diener, 2013). Schueller and Parks (2014) argued that in addition to the (positive) aim of an intervention, the pathways through which the interventions operate is a second essential component in deciding if an intervention can be considered as a PPI. They identified the following five pathways:

(1) savoring (intensifying and prolonging momentary pleasurable experiences),

(2) expressing gratitude (through reflection and activities of expression),

(3) engaging in acts of kindness,

(4) promoting positive relationships, and

(5) promoting meaning and purpose.

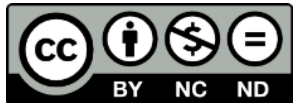

Copyright belongs to the author(s) www.internationaljournalofwellbeing.org 
More recently, Shin and Lyobomirsky (2017) used the term "positive activity interventions" instead of PPIs, and the term " positive interventions" is also used (Gander, Proyer, Ruch \& Wyss, 2013; Rashid, 2009). We define positive psychology interventions (PPIs) as interventions aiming at increasing positive feelings, behaviors and cognitions and using pathways or strategies to increase wellbeing based on theories and empirical research, following Schueller and Parks (Schueller \& Parks, 2014; Schueller, Kashdan, \& Parks, 2014).

The growth of the scientific output from scholars in the field of positive psychology is not only limited to publications from Western countries; since 2012, there has also been a strong increase in the number of studies originating from non-Western countries (Hendriks et al., $2018 b)$. Previously published meta-analyses of randomized controlled trials have examined the overall effects of PPIs. A meta-analysis by Bolier et al. (2013) that included 39 trials reported small effect sizes for subjective wellbeing, psychological wellbeing, and depression. More recently, the authors of the current meta-analysis also examined the effects of 37 multi-component PPI (MPPIs) programs containing at least three positive psychology activities (Hendriks et al., 2018b). This meta-analysis found a moderate effect size for subjective wellbeing, a small to moderate effect size for psychological wellbeing, and a small effect for depression and anxiety. In addition, this study showed that the region of origin of the studies was a strong and significant moderator. More precisely, PPIs from non-Western countries had substantially larger effects than PPIs from Western countries. For example, the effect size for subjective wellbeing was large $(g=1.13)$ for non-Western studies, compared to small $(g=0.29)$ for studies from Western countries. Effect sizes for psychological wellbeing and depression also showed substantial differences. Effect sizes for non-Western studies were three to five times larger than the effect sizes for studies from Western countries. The difference was mainly attributed to the overall lower quality of non-Western studies. It should be noted that, following Gosling, Sandy, John, and Potter (2010), North America, Western Europe, Australia, Israel, and New Zealand were classified as Western countries. A risk of bias analysis was conducted to assess the quality of the studies. Findings from this analysis demonstrated that the average quality of studies from Western countries was moderate, whereas the average quality of studies from non-Western countries was low (Hendriks et al., 2018b). For example, from the eight non-Western studies, only one used an intention-to-treat analysis for the statistical data, in only one study assessment was blinded, none described the process of randomization (sequence generation) or the allocation of participants, and all studies had fewer than 50 participants per condition.

\subsection{Present study}

Since only multi-component PPIs were include in the aforementioned meta-analysis (Hendriks et al., 2018b), findings on the efficacy of studies from non-Western countries were based on a relatively small number of studies (nine out of $37 \mathrm{RCTs}$ ). In this meta-analysis, RCTs of single component, as well as multi-component PPIs from non-Western countries will be compared to interventions performed in Western countries. The characteristics of the studies, including study quality, will also be examined, to attempt to explain differences in effect sizes.

\section{Method}

The Preferred Reporting Items for Systematic Reviews and Meta-Analyses (PRISMA) guidelines (Moher et al., 2010; Moher, Liberati, Teztlaff, \& Altman, 2009) and the recommendations of the Cochrane Back Review group (Higgins et al., 2011) were followed in the planning and the implementation of the meta-analysis. No protocol was registered. 


\subsection{Search strategy}

A systematic literature search was conducted by the first author (TH) and second author (MS) in the following databases: PubMed, PsycINFO, and Scopus, from 1998 through 2017. PubMed and PsycINFO are recommended as databases to be used in meta-analyses in mental health research (Cuijpers, 2016). We did not include the Cochrane Central Register of Controlled Trials, since that database does not allow export of citations, which complicates the screening process. Instead, we selected Scopus, another often used database for systematic reviews and metaanalyses (Davis, Mengersen, Bennett, \& Mazerolle, 2014). The search was conducted by the first and third author (TH, AH), and the last run was conducted on December, 8, 2017. Databases were searched with the following terms: "positive psycho*" OR wellbeing OR happiness OR happy OR flourishing OR "life satisfaction" OR "satisfaction with life" OR optimism OR gratitude OR strengths OR forgiveness OR compassion AND "random*". Search strings were adapted according to the database (see Appendix A). The reference lists of three meta-analyses (Bolier et al., 2013; Chakhssi, Kraiss, Sommers-Spijkerman, \& Bohlmeijer, 2018; Dickens, 2017) and seven review articles on PPIs (Casellas-Grau, Font, \& Vives, 2014; Ghosh \& Deb, 2016; Macaskill, 2016; Rashid, 2015; Sutipan, Intarakamhang, \& Macaskill, 2017; Walsh, Cassidy, \& Priebe, 2016; Woodworth, O-Brien-Malone, Diamond, \& Schüz, 2016) were also checked. In addition, a hand search through the websites of three known non-Western journals in the field of positive psychology was conducted, namely, the websites of the Indian Journal of Positive Psychology, the Iranian Journal of Positive Psychology, and the Middle East Journal of Positive Psychology. Preliminary findings of a study that was recently conducted by the authors of this meta-analysis (Hendriks et al., 2017) were also included.

\subsection{Selection of studies}

After removal of duplicates, we screened titles and abstracts. Full texts of potentially relevant articles were fully assessed. This was done by the first (TH) and third (AH) author independently. Studies were included based on the following criteria:

(1) randomized controlled trials;

(2) conducted in non-Western countries;

(3) administered to healthy adults or adults in clinical populations;

(4) published in peer reviewed journals, in the English language;

(5) validated outcome measures were used to examine the effects on subjective and psychological wellbeing, depression, or anxiety.

We excluded studies that: (1) did not provide sufficient data to calculate post-test effect sizes per condition; (2) were published in book chapters, dissertations, and studies in grey literature; (3) reported effects of mindfulness-based therapies, Acceptance and Commitment Therapy, and Compassion Focused Therapy, considering the vast number of meta-analyses on these interventions that have been published in the past decade.

\subsection{Data extraction}

The following data was gathered: author(s), year of publication, country of origin, study design, sample description, intervention type, delivery mode, description of control group, number of sessions, duration of session period, follow-up assessment, number or participants per condition at post-test level, mean age and standard deviation of participants, percentage of female participants, retention rate at post-test level, and the questionnaires that measured subjective wellbeing, psychological wellbeing, depression or anxiety. Means and standard deviations at 
post-test were extracted. In the case of insufficient data or unclear reporting in the studies, we contacted the authors through e-mail.

\subsection{Quality assessment}

The quality of the studies was independently assessed by the first (TH) and third ( $\mathrm{AH})$ author, using the Cochrane Collaboration's tool for assessing risk of bias in randomized trials (Higgins et al., 2011). The following six criteria were assessed: random sequence generation, allocation concealment, blinding of participants and personnel, description of drop-outs, power analysis, and intention-to-treat analysis or no drop-outs. One point was appointed for each criterion that was met, as described in the studies. According to this assessment tool, the quality of a study was assessed as "high" when five or six criteria were met, "moderate" when three or four criteria were met, and "low" when fewer than three criteria were met. Disagreements between the first $(\mathrm{TH})$ and third $(\mathrm{AH})$ author were discussed until consensus was reached. If any disagreement persisted, the second author (MS) or fifth author (EB) were consulted.

\subsection{Data analysis}

Data analysis was conducted with the program Comprehensive Meta-Analysis (CMA, version 3.3.070, Biostat, Inc.). Means, standard deviations, and sample sizes for each study were used to calculate the effect sizes. For each comparison between a PPI and a control group, we calculated the Hedges' adjusted $g$ because Hedges' $g$ is more accurate than Cohen's $d$ when sample sizes of the studies are small (Cuijpers, 2016). Effect sizes indicate the difference between the two groups at post-test and were calculated by subtracting the average score of the PPI group from the average score of the comparison group (both at post-test) and dividing the result by the pooled standard deviations of the two groups. Effect sizes of 0 to 0.32 can be considered as small, effect sizes of 0.33 to 0.55 as moderate, and effect sizes of 0.56 to 1.2 as large (Lipsey \& Wilson, 1993). If more than one measure was used for a similar outcome, we pooled the means and the standard deviations, so that each study outcome had one effect size (Thalheimer \& Cook, 2002). If more than one experimental group was compared to another active control group and a non-active control condition in a particular study, we used data from the active control group. Standardized mean differences (SMD) with 95\% confidence intervals (CI) were calculated as the differences in means between groups divided by the pooled standard deviation using Hedges' $g$. A positive SMD was defined as an indicator of beneficial effects for the PPI compared with the control condition. Intention-to-treat samples were used if possible. For the calculation of effect sizes for subjective wellbeing, we used instruments that explicitly indicated that they measured emotional wellbeing, as defined by Keyes (2007). These were the Mental Health Short Form - subjective wellbeing subscale (Keyes, 2005), the Oxford Happiness Inventory (Hills \& Argyle, 2002), the WHOQOL-BREF Psychological Health Scale (WhoqolGroup, 1998), the Subjective Happiness Scale (Lyubomirsky \& Lepper, 1999), the Subjective Well-being Questionnaire (Molavi, Torkan, Soltani, \& Palahang, 2010), the Life Satisfaction Index/Scale (Adams, 1969), the Satisfaction with Life Scale (Diener, Emmons, Larsen, \& Griffin, 1985), the Enright Forgiveness Inventory - positive affect subscale (Enright \& Rique, 2004), the Index of Well-Being \& Index of General Affect (Campbell, Converse, \& Rodgers, 1976), the Multiple Mood Scale - positive emotions (Terasaki, Kishimoto, \& Koga, 1992), the Positive and Negative Affect Schedule, and the Scale of Positive and Negative Experience (Watson, Clark, \& Tellegen, 1988). For psychological wellbeing, we used instruments that explicitly measured psychological wellbeing, as defined by Keyes (2007). These were the Flourishing Scale (Diener et al., 2010), the Mental Health Short Form psychological wellbeing subscale (Keyes, 2005), Well-being/Ill-being Scale (Kitwood \& Bredin, 
1997), the Ego Resilience Scale (Block \& Kremen, 1996), the Self Compassion Scale (Neff, 2003), the Symptom Checklist-90 Revised - inverted score (Derogatis \& Unger, 2010), and two unnamed scales measuring empowerment and organizational commitment (Im, Cho, Kim \& Heo, 2016). For depression and anxiety, we used instruments that explicitly measured depression and anxiety. These were the Beck Depression Index (Beck, Steer, \& Brown, 1996), the Center for Epidemiological Studies - Depression Scale (Andresen, Malmgren, Carter, \& Patrick, 1994), the Depression Anxiety Stress Scale (Lovibond \& Lovibond, 1995), the Geriatric Depression Scale (Lee, Chiu, \& Kwong, 1994), the Beck Anxiety Inventory (Beck \& Steer, 1990), Death Anxiety Scale (Templer, 1970), and the State-Trait Anxiety Inventory (Spielberger, 2010). If more than one measure for a specific outcome was reported, a variance-weighted average of effect sizes from the scales within each study was used to calculate one effect size (Marín-Martínez \& SánchezMeca, 1999). When follow-up data was available, between-group effect sizes (Hedges' $g$ ) at these time points were calculated.

\subsection{Heterogeneity}

Statistical heterogeneity between studies was tested using the $I^{2}$ statistic. This is a measure that indicates study-to-study dispersion due to real differences, over and above random sampling error (Higgins \& Thompson, 2002). A randomized effects model with a 95\% confidence interval and a two-tailed test were performed for the heterogeneity analyses. The $\mathrm{I}^{2}$ statistic was used to estimate the percentage of heterogeneity across the studies not attributable to random sampling error alone. A value of $0 \%$ indicated no heterogeneity. Values of $75 \%, 50 \%$ and $25 \%$ reflected high, moderate, and low degrees of heterogeneity, respectively (Higgins \& Thompson, 2002). Significant heterogeneity was indicated by a significant $Q$ statistic $(p \leq 0.05)$, meaning that one or more variables were present that moderated the observed effect size. All studies were included, outliers were not removed.

\subsection{Subgroup analyses}

Explanatory subgroup analyses were conducted to examine moderating effects of seven possible moderators. These moderators were:

(1) study population: clinical or non-clinical;

(2) mode of delivery of the PPI: group or self-help;

(3) intervention type: single component or multi-component;

(4) type of control group: active/placebo or non-active/waitlist;

(5) duration of the intervention: $\leq 8$ weeks or $>8$ weeks;

(6) cultural adaptation of the PPI: yes or no;

(7) quality rating of the study: low, moderate, or high.

\subsection{Publication bias}

Publication bias was assessed in the following ways. First, a funnel plot was created by plotting the overall mean effect size against study size. Absence of publication bias is present when there is a symmetrical distribution of studies around the effect size (Sterne, Egger, \& Moher, 2008). Second, a fail-safe $\mathrm{N}$ was calculated for each analysis to test the asymmetry of each funnel plot. The fail-safe $\mathrm{N}$ indicates the number of unpublished non-significant studies that would be required to lower the overall effect size below significance (Egger, Smith, Schneider, \& Minder, 1997; Orwin, 1983). Findings were considered robust if the fail-safe $N \geq 5 k+10$, where $k$ is the number of studies (Rosenberg, 2005). Third, the Trim and Fill method (Duval \& Tweedie, 2000) 
was used. This procedure imputes the effect sizes of missing studies and produces an adjusted effect size accounting for the missing studies.

\section{Results}

\subsection{Study selection}

We identified a total of 8,172 records. After removal of duplicates, 7,516 records remained. These records were screened, after which 372 records remained. These articles were assessed for eligibility, and finally 28 studies were included in the meta-analysis, which were published in 29 articles. The complete selection process is shown in Figure 1.

\section{Figure 1. Flow diagram of the selection process of RCTs on PPIs from non-Western countries}
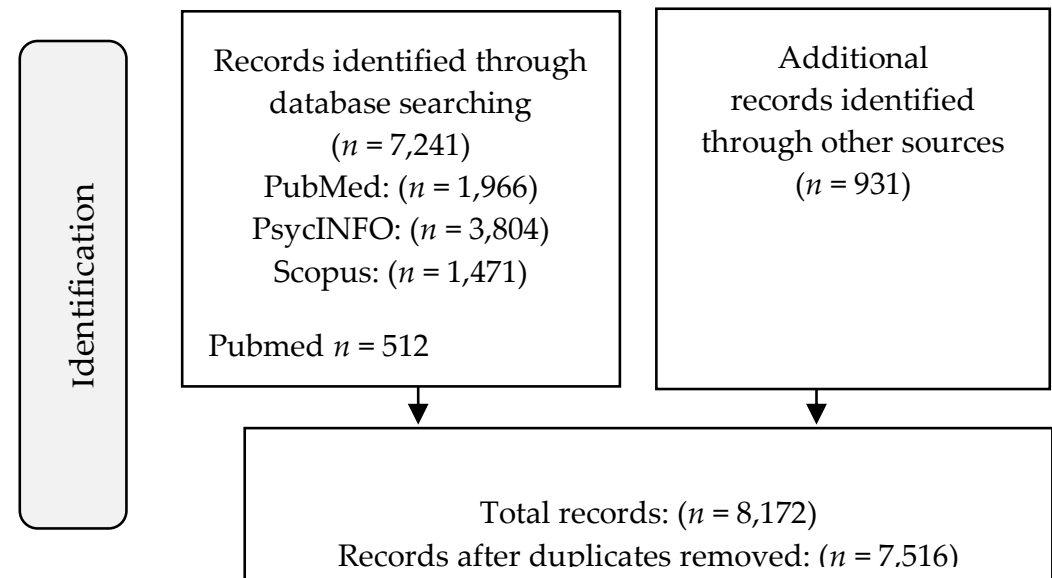

Total records: $(n=8,172)$

Records after duplicates removed: $(n=7,516)$
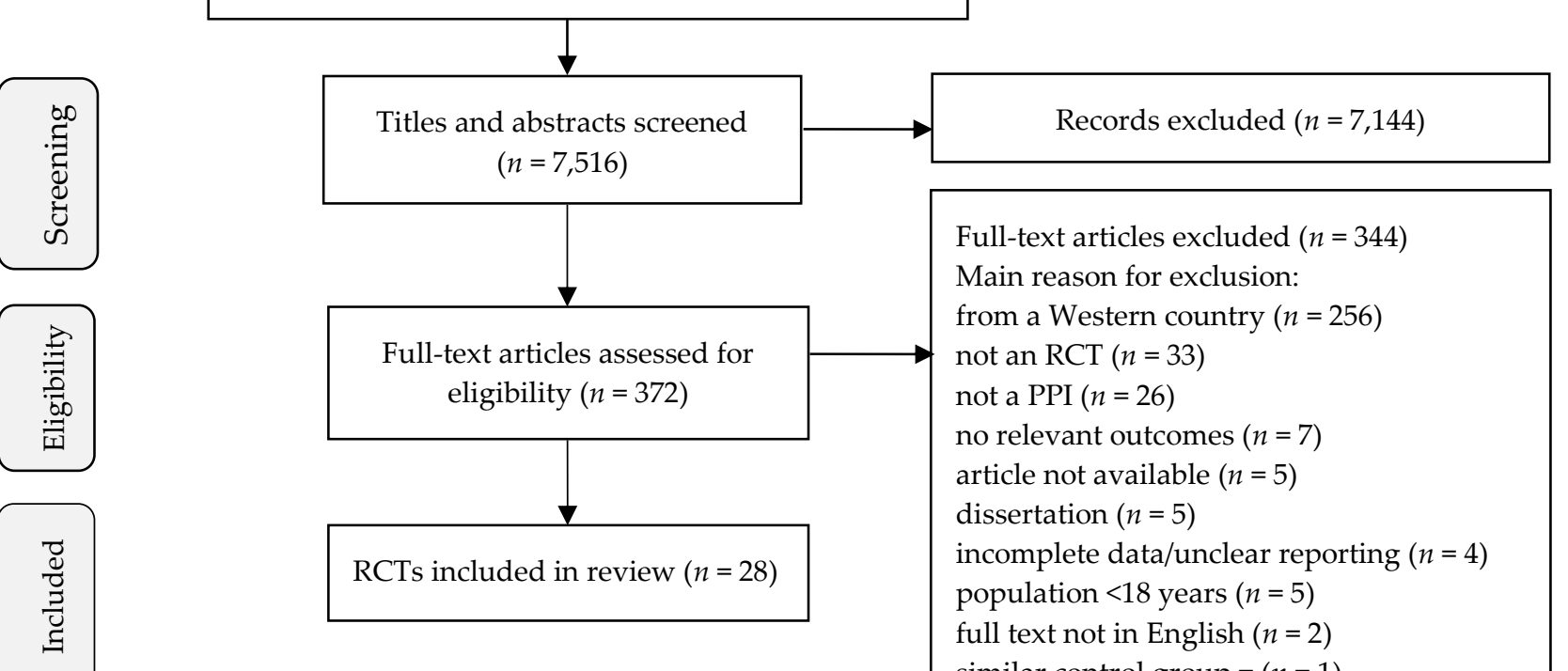

records identified through other sources

$(n=931)$

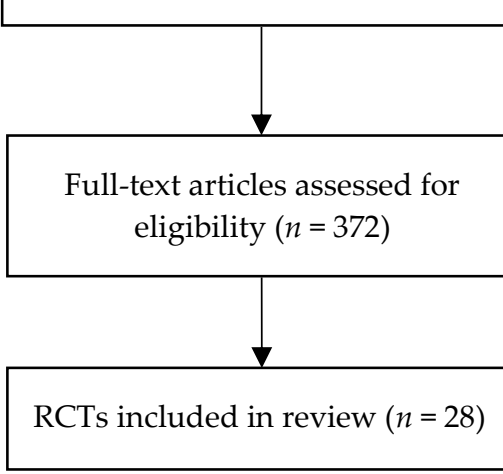

Full-text articles excluded $(n=344)$

Main reason for exclusion:

from a Western country $(n=256)$

not an RCT $(n=33)$

not a PPI $(n=26)$

no relevant outcomes $(n=7)$

article not available $(n=5)$

dissertation $(n=5)$

incomplete data/unclear reporting $(n=4)$

population $<18$ years $(n=5)$

full text not in English $(n=2)$

similar control group $=(n=1)$ 


\subsection{Study characteristics}

The RCTs were published between 2012 and December 2017. Seven studies (25\%) were conducted among clinical populations and 21 studies (75\%) among non-clinical populations. Delivery modes were group based $(n=21,75 \%)$ and self-help $(n=7,25 \%)$. Eleven studies (39\%) had an active control group and 17 studies $(61 \%)$ had a non-active control group (waiting-list, $n$ $=1$; no intervention, $n=16$ ). The studies included a total of 3,009 participants.

Sample sizes from the intervention groups ranged from nine to 828, with a mean of 25.7 participants (excluding the cluster randomized controlled trial). Fourteen studies $(50 \%)$ had fewer than 20 participants in the intervention group, ten (36\%) had between 20-40 participants in the intervention group, three (11\%) had between $40-60$ participants in the intervention group, and there was one (4\%) cluster randomized controlled trial with 828 participants in the intervention group. Twelve (43\%) studies were from China (five from mainland China, seven from Hong Kong), six (21\%) from Iran, three (11\%) from Taiwan, two (7\%) from Japan, two (7\%) from South Korea, and Malaysia, Suriname, and Turkey each accounted for one study. One of the self-help-based interventions was an online intervention. Twenty-five studies reported the percentage of female participants, which was $67 \%(n=2,010)$. Excluding one cluster randomized controlled trial lowered the percentage of female participants to $61 \%(n=1,817)$. Twenty-one studies measured subjective wellbeing (SWB), eight studies measured psychological wellbeing (PWB), 13 studies measured depression, and five studies reported on anxiety. All main characteristics of the studies are presented in Table 1 (below).

\subsection{Quality assessment}

In total, the six quality criteria were assessed for 28 studies. The lowest score was 0 (five studies), the highest score was 6 (two studies). The overall study quality was low, with a mean score of $1.79(\mathrm{SD}=1.67)$. Three studies $(11 \%)$ were rated with a high quality, two $(7 \%)$ with a moderate quality, and $23(82 \%)$ with a low quality. The description of the method that was used to generate the allocation sequence (sequence generation) was reported in 10 studies (36\%). Description of the method used to conceal the allocation sequence (allocation concealment) was reported in only five studies (18\%). Blinding of main outcome assessment was described in only three studies (11\%). In 19 studies (68\%) it was clearly described how many drop-outs there were during the intervention period. A power-analysis was conducted in seven studies (25\%). Five studies (18\%) used an intention-to-treat analysis, and one study (4\%) reported zero drop-outs. The outcome of the quality assessment is shown in Table 2 (below).

\subsection{Post-test treatment effects}

The random effects model showed that PPIs were significantly more effective for all outcome measures compared to the control conditions. The main results are presented in Table 3 (below) and explained below. Effect sizes of the individual studies are plotted in Figures 2, 3, and 4 (below). 
Table 1a. Study characteristics of RCTs on PPI from non-Western countries

\begin{tabular}{|c|c|c|c|c|c|c|c|c|c|c|c|c|}
\hline First author/ year/country & Design & Sample & Intervention & Delivery & Control group & $\begin{array}{l}\text { Sessions, } \\
\text { duration }\end{array}$ & $\begin{array}{l}\text { Follow } \\
\text { up }\end{array}$ & $\begin{array}{l}\mathbf{N} \\
\text { (post) }\end{array}$ & $\begin{array}{l}\text { Mean } \\
\text { /SD } \\
\end{array}$ & $\begin{array}{l}\% \\
\text { Female }\end{array}$ & $\begin{array}{l}\text { Retention } \\
\text { rate (post) }\end{array}$ & Outcome measures \\
\hline Al-Seheel, 2016, Iran & RCT & Students & $\begin{array}{l}\text { Gratitude, } \\
\text { Islamic-based }\end{array}$ & Self-help & PPI + Placebo & $2 \mathrm{w}$ & - & $\begin{array}{l}\mathrm{Ne}=19 \\
\mathrm{Nc} 1=20 \\
\mathrm{Nc} 2=21\end{array}$ & $21.9(1.2)$ & $85 \%$ & $\mathrm{Nt}=95 \%$ & SWB: SPANE, SWLS \\
\hline Arimitsu, 2016, Japan & $\mathrm{RCT}$ & Healthy adults & Self-compassion & Group & Wait-list & $7,7 \mathrm{w}$ & $3 \mathrm{~m}$ & $\begin{array}{l}\mathrm{Ne}=16 \\
\mathrm{Nc}=12\end{array}$ & 23.3 & $85 \%$ & $\begin{array}{l}\mathrm{Ne}=80 \% \\
\mathrm{Nc}=60 \%\end{array}$ & $\begin{array}{l}\text { SWB: MMS, PWB: SCS } \\
\text { Dep: BDI, Anx: STAI }\end{array}$ \\
\hline Asgharipoor, 2012, Iran & $\mathrm{RCT}$ & $\begin{array}{l}\text { Patients with major } \\
\text { depression }\end{array}$ & PPT & Group & CBT & $6,12 w$ & - & $\begin{array}{l}\mathrm{Ne}=9 \\
\mathrm{Nc}=9\end{array}$ & $26.4(5.9)$ & $72 \%$ & $\begin{array}{l}\mathrm{Ne}=100 \% \\
\mathrm{Nc}=100 \%\end{array}$ & $\begin{array}{l}\text { SWB: OHI } \\
\text { PWB: SWS-PWB subscale } \\
\text { Dep: BDI }\end{array}$ \\
\hline Asl, 2014, 2016, Iran & RCT & Infertile women & PPT & Group & Wait-list & $6,6 \mathrm{w}$ & - & $\begin{array}{l}\mathrm{Ne}=15 \\
\mathrm{Nc}=16\end{array}$ & $30.5(5.7)$ & $100 \%$ & $\begin{array}{l}\mathrm{Ne}=83 \% \\
\mathrm{Nc}=89 \%\end{array}$ & $\begin{array}{l}\text { SWB: OHI } \\
\text { Dep: BDI }\end{array}$ \\
\hline Chan, 2013, China, HK & RCT & Students & $\begin{array}{l}\text { Counting } \\
\text { blessings }\end{array}$ & Self-help & Placebo & $1,8 \mathrm{w}$ & - & $\begin{array}{l}\mathrm{Ne}=40 \\
\mathrm{Nc}=41\end{array}$ & $33.7(7.2)$ & $81 \%$ & $\mathrm{Nt}=97 \%$ & SWB: SWLS \\
\hline Cheng, 2015, China, HK & $\mathrm{RCT}$ & Healthy adults & Gratitude & Self-help & Placebo & $4 w$ & $3 \mathrm{~m}$ & $\begin{array}{l}\mathrm{Ne}=34 \\
\mathrm{Nc} 1=34\end{array}$ & - & $55 \%$ & & Dep: CES-D \\
\hline Chiang, 2008, Taiwan & $\mathrm{RCT}$ & Elderly & Life review & Group & Wait-list & $8,8 w$ & - & $\begin{array}{l}\mathrm{Ne}=36 \\
\mathrm{Nc}=39\end{array}$ & $78.1(3.7)$ & $100 \%$ & $\mathrm{Nt}=71 \%$ & SWB: LISA \\
\hline Chiang, 2010, Taiwan & $\mathrm{RCT}$ & Elderly & Reminiscence & Group & Wait-list & $8,8 w$ & $3 \mathrm{~m}$ & $\begin{array}{l}\mathrm{Ne}=45 \\
\mathrm{Nc}=47\end{array}$ & $77.2(4.0)$ & $100 \%$ & $\mathrm{Nt}=75 \%$ & $\begin{array}{l}\text { PWB: SCL-90R } \\
\text { Dep: BDI }\end{array}$ \\
\hline Choy, 2016, China, HK & $\mathrm{RCT}$ & Elderly & Reminiscence & Group & Wait-list & $6,6 w$ & $6 w$ & $\begin{array}{l}\mathrm{Ne}=39 \\
\mathrm{Nc}=42\end{array}$ & $78.0(7.1)$ & $67 \%$ & $\begin{array}{l}\mathrm{Ne}=85 \% \\
\mathrm{Nc}=62 \%\end{array}$ & $\begin{array}{l}\text { SWB: LSS } \\
\text { Dep: GDS }\end{array}$ \\
\hline Deng, China, 2016 & RCT & Healthy adults & Gratitude & Group & No intervention & $5 w$ & - & $\begin{array}{l}\mathrm{Ne}=36 \\
\mathrm{Nc}=29\end{array}$ & $35.7(9.4)$ & $0 \%$ & - & SWB: SWBQ \\
\hline Dowlatabadi, 2016, Iran & RCT & $\begin{array}{l}\text { Women with } \\
\text { breast cancer }\end{array}$ & PPT & Group & No intervention & $10,10 w$ & - & $\begin{array}{l}\mathrm{Ne}=17 \\
\mathrm{Nt}=17\end{array}$ & $36.6(5.5)$ & $100 \%$ & $\begin{array}{l}\mathrm{Ne}=76 \% \\
\mathrm{Nc}=81 \%\end{array}$ & $\begin{array}{l}\text { SWB: OHI } \\
\text { Dep: BDI }\end{array}$ \\
\hline Guo, 2016, China, HK & RCT & Students & PPT & Group & No intervention & $8,8 \mathrm{w}$ & $3 \mathrm{~m}$ & $\begin{array}{l}\mathrm{Ne}=34 \\
\mathrm{Nc}=42\end{array}$ & $20.4(1.2)$ & $95 \%$ & $\begin{array}{l}\mathrm{Ne}=81 \% \\
\mathrm{Nc}=98 \%\end{array}$ & Dep: BDI \\
\hline Hendriks, 2017, Suriname & $\mathrm{RCT}$ & Healthy adults & MPPI & Group & Wait-list & $7,7 w$ & - & $\begin{array}{l}\mathrm{Ne}=80 \\
\mathrm{~N} 1=72\end{array}$ & $36.3(9.6)$ & $60 \%$ & $\begin{array}{l}\mathrm{Ne}=91 \% \\
\mathrm{Nc}=91 \%\end{array}$ & $\begin{array}{l}\text { SWB, PWB: MHSF } \\
\text { Dep, Anx, Stress: DASS-21 }\end{array}$ \\
\hline
\end{tabular}


Table 1b. Study characteristics of RCTs on PPI from non-Western countries

\begin{tabular}{|c|c|c|c|c|c|c|c|c|c|c|c|c|}
\hline First author/ year/country & Design & Sample & Intervention & Delivery & Control group & $\begin{array}{l}\text { Sessions, } \\
\text { duration }\end{array}$ & $\begin{array}{l}\text { Follow } \\
\text { up }\end{array}$ & $\begin{array}{l}\mathrm{N} \\
\text { (post) }\end{array}$ & $\begin{array}{l}\text { Mean } \\
\text { /SD } \\
\end{array}$ & $\begin{array}{l}\% \\
\text { Female }\end{array}$ & $\begin{array}{l}\text { Retention } \\
\text { rate (post) }\end{array}$ & Outcome measures \\
\hline Ho, 2016, China, HK & CRCT & Healthy families & MPPI & $\begin{array}{l}\text { Group } \\
\text { Self-help }\end{array}$ & Placebo & $2,4 \mathrm{w}$ & $12 \mathrm{w}$ & $\begin{array}{l}\mathrm{Ne} 1=828 \\
\mathrm{Ne} 2=433\end{array}$ & - & $75 \%$ & $\begin{array}{l}\mathrm{Ne}=71 \% \\
\mathrm{Nc}=83 \%\end{array}$ & SWB: SHS \\
\hline Hwang, 2016, China* & $\mathrm{RCT}$ & Student & PPT & Group & No active control & $10,5 w$ & $4 \mathrm{~m}$ & $\begin{array}{l}\mathrm{Ne}=8 \\
\mathrm{Nc} 1=8 \\
\mathrm{Nc} 2=8\end{array}$ & $22.7(2.3)$ & $67 \%$ & $\begin{array}{l}\mathrm{Ne}=72 \% \\
\mathrm{Nc} 1 / 2= \\
80.0 \%\end{array}$ & $\begin{array}{l}\text { SWB: SPANE } \\
\text { PWB: FS }\end{array}$ \\
\hline Im, 2016, Korea & RCT & Nurses & MPPI & Group & No intervention & $4,9 w$ & - & $\begin{array}{l}\mathrm{Ne}=25 \\
\mathrm{Nc}=24\end{array}$ & $25.6(2.7)$ & $82 \%$ & $\begin{array}{l}\mathrm{Ne}=100 \% \\
\mathrm{Nc}=96 \%\end{array}$ & $\begin{array}{l}\text { PWB: ERS, empowerment, } \\
\text { work commitment }\end{array}$ \\
\hline Ji, 2016, China & RCT & Students & Forgiveness & Group & Wait-list & $10,10 w$ & & $\begin{array}{l}\mathrm{Ne}=16 \\
\mathrm{Nc}=12\end{array}$ & $20.2(1.4)$ & $89 \%$ & $\mathrm{Nt}=78 \%$ & $\begin{array}{l}\text { SWB: EFI } \\
\text { Anx: STAI }\end{array}$ \\
\hline Khayatan, 2014, Iran & $\mathrm{RCT}$ & $\begin{array}{l}\text { Women } \\
\text { with MS }\end{array}$ & PPT & Group & No intervention & $6,6 w$ & & $\begin{array}{l}\mathrm{Ne}=15 \\
\mathrm{Nc}=15\end{array}$ & $31.1(6.5)$ & $100 \%$ & $\begin{array}{l}\mathrm{Ne}=100 \% \\
\mathrm{Nc}=100 \%\end{array}$ & Dep: BDI \\
\hline Koydemir, 2015, Turkey* & RCT & Students & $\begin{array}{l}\text { Strength-based } \\
\text { intervention }\end{array}$ & $\begin{array}{l}\text { Self-help } \\
\text { (online) }\end{array}$ & Wait-list & $5,8 w$ & & $\begin{array}{l}\mathrm{Ne}=44 \\
\mathrm{Nc}=36\end{array}$ & $18.7(1.0)$ & $48 \%$ & - & SWB: SHS, SWLS, PHS \\
\hline Lai, 2004, China & $\mathrm{RCT}$ & Elderly & Reminiscence & Group & Active control & $6,6 w$ & $6 w$ & $\begin{array}{l}\mathrm{Ne}=36 \\
\mathrm{Nc} 1=30\end{array}$ & $85.6(7.0)$ & $68 \%$ & $\mathrm{Nt}=85 \%$ & PWB: WIB \\
\hline Lau, 2011, China & $\mathrm{RCT}$ & Elderly & Gratitude & Group & $\begin{array}{l}\text { Active control, } \\
\text { No intervention }\end{array}$ & 1 & & $\begin{array}{l}\mathrm{Ne}=29 \\
\mathrm{Nc} 1=25 \\
\mathrm{Nc} 2=29\end{array}$ & $62.5(7.1)$ & $60 \%$ & - & ANX: Das \\
\hline Lü, 2013, China & $\mathrm{RCT}$ & Students & MPPI & Group & No intervention & $8,8 w$ & & $\begin{array}{l}\mathrm{Ne}=16 \\
\mathrm{Nc}=18\end{array}$ & $20.0(4.3)$ & $57 \%$ & $\begin{array}{l}\mathrm{Ne}=84 \% \\
\mathrm{Nc}=100 \%\end{array}$ & SWB: PANAS \\
\hline Nikrahan, 2016, Iran & $\mathrm{RCT}$ & Healthy adults & MPPI & Group & $3 \times$ PPI, Wait-list & $6,6 w$ & $15 w$ & $\begin{array}{l}\mathrm{Ne} 1=13 \\
\mathrm{Ne} 2=13 \\
\mathrm{Ne} 3=15 \\
\mathrm{Nc}=14\end{array}$ & $56.6(8.7)$ & $24 \%$ & $\mathrm{Nt}=100 \%$ & $\begin{array}{l}\text { SWB: OHI } \\
\text { Dep: BDI }\end{array}$ \\
\hline Otsuka, 2012 Japan & $\mathrm{RCT}$ & Healthy adults & Gratitude & Self-help & Placebo & $4 w$ & $1 \mathrm{~m}$ & $\begin{array}{l}\mathrm{Ne}=19 \\
\mathrm{Nc}=19\end{array}$ & $48.5(5.1)$ & $28 \%$ & $\mathrm{Nt}=50 \%$ & SWB: SHS \\
\hline
\end{tabular}


Table 1c. Study characteristics of RCTs on PPI from non-Western countries

\begin{tabular}{|c|c|c|c|c|c|c|c|c|c|c|c|c|}
\hline First author/ year/country & Design & Sample & Intervention & Delivery & Control group & $\begin{array}{l}\text { Sessions, } \\
\text { duration }\end{array}$ & $\begin{array}{l}\text { Follow } \\
\text { up }\end{array}$ & $\begin{array}{l}\mathbf{N} \\
\text { (post) }\end{array}$ & $\begin{array}{l}\text { Mean } \\
\text { /SD }\end{array}$ & $\begin{array}{l}\% \\
\text { Female }\end{array}$ & $\begin{array}{l}\text { Retention } \\
\text { rate (post) }\end{array}$ & Outcome measures \\
\hline Wong, 2016, China & RCT & Students & $\begin{array}{l}\text { Self-compassion } \\
\text { writing }\end{array}$ & Self-help & Placebo & $\begin{array}{l}3 \mathrm{~d}, \\
3 \mathrm{~m}\end{array}$ & & $\begin{array}{l}\mathrm{Ne}=33 \\
\mathrm{Nc}=32\end{array}$ & $20.5(1.4)$ & $54 \%$ & $\mathrm{Nt}=100 \%$ & Dep: CES-D \\
\hline Wu, 2016, Taiwan & $\mathrm{RCT}$ & $\begin{array}{l}\text { Patients } \\
\text { with dementia }\end{array}$ & $\begin{array}{l}\text { Spiritual } \\
\text { reminiscence }\end{array}$ & Group & No intervention & $6,6 \mathrm{w}$ & & $\begin{array}{l}\mathrm{Ne}=50 \\
\mathrm{Nc}=53\end{array}$ & $73.6(7.4)$ & $69 \%$ & $\begin{array}{l}\mathrm{Ne}=100 \% \\
\mathrm{Nc}=94 \%\end{array}$ & SWB: LISA \\
\hline Yousefi, 2015, Iran & $\mathrm{RCT}$ & Elderly women & Reminiscence & Group & Active control & $6,3 \mathrm{w}$ & $1 \mathrm{~m}$ & $\begin{array}{l}\mathrm{Ne}=14 \\
\mathrm{Nc}=14\end{array}$ & $65.2(6.4)$ & $100 \%$ & $\begin{array}{l}\mathrm{Ne}=93 \% \\
\mathrm{Nc}=88 \%\end{array}$ & SWB: OHQ \\
\hline Zhang, 2014, China & $\mathrm{RCT}$ & Students & Forgiveness & Group & No intervention & 1 & $4 \mathrm{w}$ & $\begin{array}{l}\mathrm{Ne}=10 \\
\mathrm{Nc} 1=11\end{array}$ & $\begin{array}{l}22.1 \\
(1.0)\end{array}$ & $100 \%$ & $\mathrm{Nt}=100 \%$ & $\begin{array}{l}\text { SWB: IWB/IGA } \\
\text { Dep: BDI } \\
\text { Anx: BAI }\end{array}$ \\
\hline
\end{tabular}

CRCT: cluster randomized controlled trial; HK: Hong Kong; MPPI: multi-component positive psychology intervention; PPT: positive psychotherapy.

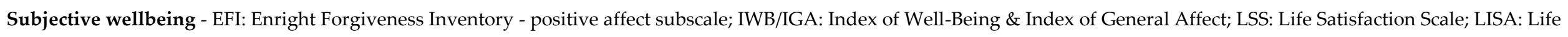

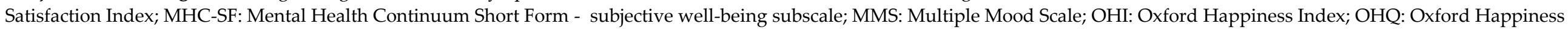
Questionnaire; PANAS: Positive and Negative Affect Schedule ; PHS: WHOQOL-BREF Psychological Health Scale; SHS: Subjective Happiness Scale; SPANE: Scale of Positive and Negative Experience; SWBQ: Subjective Well-being Questionnaire; SWLS: Satisfaction With Life Scale; SWS: Subjective Wellbeing Scale

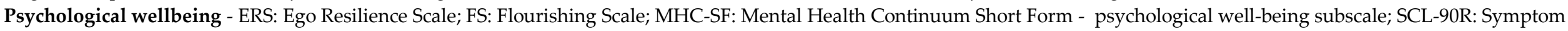

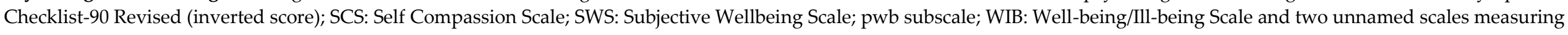
empowerment and organizational commitment

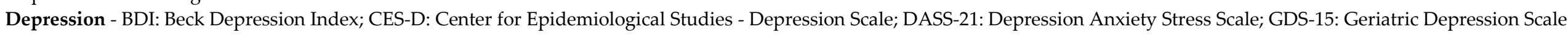
Anxiety - BAI: Beck Anxiety Inventory; DAS: Death Anxiety Scale; DASS-21: Depression Anxiety Stress Scales; STAI: State- Trait Anxiety Inventory 
Table 2. Quality assessment of RCTs on PPIs in non-Western countries

\begin{tabular}{|c|c|c|c|c|c|c|c|c|}
\hline Studies & SG & AC & BOA & DDO & $\begin{array}{c}\mathrm{N}>50 \\
\mathrm{PA}\end{array}$ & $\begin{array}{l}\text { ITT/ } \\
0 \text { DO }\end{array}$ & $\begin{array}{l}\text { Total } \\
\text { Score }\end{array}$ & $\begin{array}{l}\text { Quality } \\
\text { Rating }\end{array}$ \\
\hline Al-Seheel, 2016 & 0 & 0 & 0 & 1 & 0 & 1 & 2 & Low \\
\hline Arimitsu, 2016 & 0 & 0 & 0 & 1 & 0 & 0 & 1 & Low \\
\hline Asgharipoor, 2012 & 0 & 0 & 0 & 0 & 0 & 0 & 0 & Low \\
\hline Asl, 2014, 2016 & 0 & 0 & 0 & 1 & 0 & 0 & 1 & Low \\
\hline Chan, 2013 & 0 & 0 & 1 & 1 & 0 & 0 & 2 & Low \\
\hline Cheng, 2015 & 1 & 1 & 1 & 1 & 1 & 1 & 6 & High \\
\hline Chiang, 2008 & 1 & 0 & 0 & 0 & 0 & 0 & 1 & Low \\
\hline Chiang, 2010 & 1 & 0 & 0 & 1 & 0 & 0 & 2 & Low \\
\hline Choy, 2015 & 0 & 0 & 0 & 0 & 0 & 1 & 1 & Low \\
\hline Deng, 2016 & 0 & 0 & 0 & 0 & 0 & 0 & 0 & Low \\
\hline Dowlatabadi, 2016 & 0 & 0 & 0 & 1 & 0 & 0 & 1 & Low \\
\hline Guo, 2016 & 0 & 0 & 0 & 1 & 1 & 0 & 2 & Low \\
\hline Hendriks, 2017 & 1 & 1 & 1 & 1 & 1 & 1 & 6 & High \\
\hline Ho, 2016 & 1 & 1 & 0 & 1 & 1 & 1 & 5 & High \\
\hline Hwang, 2016 & 1 & 0 & 0 & 1 & 0 & 0 & 2 & Low \\
\hline Im, 2016 & 1 & 0 & 0 & 1 & 1 & 0 & 3 & Moderate \\
\hline Ji, 2016 & 0 & 0 & 0 & 1 & 0 & 0 & 1 & Low \\
\hline Khayatan, 2014 & 0 & 0 & 0 & 0 & 0 & 0 & 0 & Low \\
\hline Koydemir, 2015 & 0 & 0 & 0 & 0 & 0 & 0 & 0 & Low \\
\hline Lai, 2004 & 0 & 0 & 0 & 1 & 0 & 1 & 2 & Low \\
\hline Lau, 2011 & 0 & 0 & 0 & 0 & 1 & 0 & 1 & Low \\
\hline Lü, 2013 & 0 & 0 & 0 & 1 & 0 & 0 & 1 & Low \\
\hline Nikrahan, 2016 & 1 & 1 & 0 & 1 & 0 & 1 & 4 & Moderate \\
\hline Otsuka, 2006 & 0 & 0 & 0 & 1 & 0 & 0 & 1 & Low \\
\hline Wong, 2016 & 0 & 1 & 0 & 1 & 0 & 0 & 2 & Low \\
\hline Wu, 2016 & 1 & 0 & 0 & 0 & 0 & 0 & 1 & Low \\
\hline Yousefi, 2015 & 1 & 0 & 0 & 1 & 0 & 0 & 2 & Low \\
\hline Zhang, 2014 & 0 & 0 & 0 & 0 & 0 & 0 & 0 & Low \\
\hline
\end{tabular}

$\mathrm{SG}=$ Sequence generation; $\mathrm{AC}=$ Allocation concealment; $\mathrm{BOA}=$ Blinding of main outcome assessment; $\mathrm{DDO}=$ Description of drop-outs; $\mathrm{N}>50, \mathrm{PA}=\mathrm{N}>50$ or power analysis; ITT $=$ Intention-to-treat analysis or 0 drop-outs

\subsubsection{Effects on subjective wellbeing}

For subjective wellbeing, a significant moderate to large effect was observed from 21 comparisons at post-test ( $g=0.48,95 \%$ CI: 0.24 to $0.72, p<0.001)$. The effect sizes of the studies ranged from 0.34 to 2.22 . Heterogeneity analysis revealed a significant and high level of heterogeneity $\left(\mathrm{I}^{2}=80.69\right.$, Q: 103.60, $\left.p<0.001\right)$. Removing four outliers reduced the effect size to $g=0.36$ (95\% CI: 0.18 to $0.53, p<0.001)$. The heterogeneity was moderate after outliers were removed $\left(\mathrm{I}^{2}=44.22, \mathrm{Q}=28.69, p<0.001\right)$, which means that there may be methodological issues which could lead to a high risk of bias. The forest plot in Figure 2 (below) displays the post-treatment effects, including outliers. 
Table 3. Between-group effects

\begin{tabular}{|c|c|c|c|c|c|c|c|}
\hline \multirow[t]{2}{*}{ Outcome measures } & \multirow[t]{2}{*}{ Ncomp } & \multirow{2}{*}{$\begin{array}{c}\text { Hedges' } \\
\quad g\end{array}$} & \multirow[t]{2}{*}{$95 \% \mathrm{CI}$} & \multirow[t]{2}{*}{$\mathrm{Z}$} & \multicolumn{2}{|c|}{ Heterogeneity } & \multirow{2}{*}{$\begin{array}{c}\text { Fail-safe } \\
\mathbf{N}\end{array}$} \\
\hline & & & & & $Q$ value & $\mathrm{I}^{2}$ & \\
\hline \multicolumn{8}{|l|}{ All studies post-treatment } \\
\hline Subjective wellbeing & 21 & 0.48 & $(0.24-0.72)$ & $3.97^{* * *}$ & $103.60^{* * *}$ & 80.69 & 272 \\
\hline Psychological wellbeing & 8 & 0.40 & $(-0.03-0.83)$ & $1.81^{* *}$ & $35.26^{* * *}$ & 80.15 & 29 \\
\hline Depression & 13 & 0.62 & $(0.19-1.05)$ & $2.81^{* *}$ & $94.87^{* * *}$ & 87.35 & 202 \\
\hline Anxiety & 5 & 0.95 & $(0.28-1.61)$ & $2.77^{* *}$ & $22.95^{* * *}$ & 82.57 & 41 \\
\hline \multicolumn{8}{|l|}{$\begin{array}{l}\text { Studies post-treatment, } \\
\text { excl. outliers }\end{array}$} \\
\hline Subjective wellbeing & 17 & 0.36 & $(0.18-0.53)$ & $4.01^{* * *}$ & $28.69^{* * *}$ & 44.22 & - \\
\hline Psychological wellbeing & 7 & 0.22 & $(-0.05-0.49)$ & $1.61 \mathrm{~ns}$ & $9.74 \mathrm{~ns}$ & 38.37 & - \\
\hline Depression & 10 & 0.69 & $(0.38-0.99)$ & $4.44^{* * *}$ & $26.55^{* * *}$ & 66.11 & - \\
\hline \multicolumn{8}{|l|}{$\begin{array}{l}\text { Follow-up effects, } \\
\text { excl. outliers }\end{array}$} \\
\hline Subjective wellbeing & 8 & 0.43 & $(0.08-0.77)$ & $2.45^{*}$ & $21.61^{* *}$ & 67.60 & - \\
\hline Depression & 7 & 0.77 & $(0.23-1.30)$ & $2.82^{* * *}$ & $38.55^{* *}$ & 84.43 & - \\
\hline
\end{tabular}

${ }^{*} p<0.05 ;{ }^{* *} p<0.01 ;{ }^{* * *} p<0.001 ;$ ns: non-significant

\subsubsection{Effects on psychological wellbeing}

For psychological wellbeing (eight comparisons), a significant moderate effect was observed ( $g=0.40,95 \%$ CI: -0.05 to $0.83, p=0.070)$ at post-treatment. Effect sizes ranged from -0.42 to 1.50. Heterogeneity was significant and high $\left(\mathrm{I}^{2}=80.15, \mathrm{Q}=35.26, p<0.001\right)$. After excluding one outlier, significant effects were no longer found. Figure 3 (below) displays the posttreatment effects in a forest plot.

\subsubsection{Effects on depression}

A significant large effect for depression (13 comparisons) was observed ( $g=0.62,95 \%$ CI: 0.19 to $1.05, p<0.001)$ at post-treatment. Effect sizes of studies ranged from -0.89 to 2.45 . Heterogeneity was significant and high $\left(\mathrm{I}^{2}=87.35, \mathrm{Q}=94.87, p=0.000\right)$. Removing three outliers increased the effect size $(g=0.68,95 \%$ CI: 0.38 to $0.99, p<0.001)$ and heterogeneity was significant and high $\left(\mathrm{I}^{2}=66.11, \mathrm{Q}=26.55, p<0.01\right)$. The forest plot in Figure 4 (below) displays the post-treatment effects. 
Figure 2. Effects of PPIs on subjective wellbeing, including outliers

Studyname

Al-Saheel, 2016

Arimitsu, 2016

Asgharipoor, 2012

Asl, 2014

Chan, 2013

Chiang, 2008

Choy, 2015

Deng, 2016

Dowlatabadi, 2016

Hendrils, 2017

Ho, 2016

Hwang, 2016

Ji, 2016

Koydemir, 2015

Lï, 2013

Nikrahan, 2013

Otsuka, 2012

Wong, 2016

Wu, 2016

Yousefi, 2015

Zhang, 2014

\begin{tabular}{|c|c|c|c|c|c|c|}
\hline \multirow[b]{2}{*}{$\begin{array}{l}\text { Hedges's } \\
\text { g }\end{array}$} & \multirow[b]{2}{*}{$\begin{array}{l}\text { Standard } \\
\text { error }\end{array}$} & \multicolumn{3}{|c|}{ Statistics for each study } & \multirow[b]{2}{*}{ Z-Value } & \multirow[b]{2}{*}{ p-Value } \\
\hline & & Variance & $\begin{array}{l}\text { Lower } \\
\text { limit }\end{array}$ & $\begin{array}{l}\text { Upper } \\
\text { limit }\end{array}$ & & \\
\hline 0.19 & 0.31 & 0.10 & -0.42 & 0.81 & 0.61 & 0.54 \\
\hline 0.08 & 0.33 & 0.11 & -0.57 & 0.73 & 0.24 & 0.81 \\
\hline 1.77 & 0.54 & 0.29 & 0.72 & 2.83 & 3.30 & 0.00 \\
\hline 0.89 & 0.34 & 0.12 & 0.22 & 1.56 & 260 & 0.01 \\
\hline 0.38 & 0.23 & 0.05 & -0.06 & 0.83 & 1.69 & 0.09 \\
\hline 0.81 & 0.24 & 0.06 & 0.34 & 1.27 & 3.38 & 0.00 \\
\hline 0.15 & 0.22 & 0.05 & -0.28 & 0.59 & 0.70 & 0.48 \\
\hline 0.79 & 0.26 & 0.07 & 0.29 & 1.29 & 3.08 & 0.00 \\
\hline 1.80 & 0.41 & 0.16 & 1.01 & 2.60 & 4.44 & 0.00 \\
\hline 0.37 & 0.16 & 0.03 & 0.06 & 0.69 & 2.35 & 0.02 \\
\hline-0.08 & 0.06 & 0.00 & -0.19 & 0.04 & -1.30 & 0.19 \\
\hline-0.44 & 0.48 & 0.23 & -1.38 & 0.50 & -0.92 & 0.36 \\
\hline 0.95 & 0.39 & 0.15 & 0.18 & 1.72 & 2.43 & 0.02 \\
\hline 0.75 & 0.23 & 0.05 & 0.30 & 1.20 & 3.25 & 0.00 \\
\hline 2.22 & 0.43 & 0.18 & 1.37 & 3.06 & 5.16 & 0.00 \\
\hline 0.31 & 0.31 & 0.09 & -0.29 & 0.91 & 1.01 & 0.31 \\
\hline 0.00 & 0.28 & 0.08 & -0.56 & 0.56 & 0.01 & 1.00 \\
\hline-0.22 & 0.25 & 0.06 & -0.71 & 0.26 & -0.91 & 0.36 \\
\hline 0.43 & 0.20 & 0.04 & 0.04 & 0.81 & 2.16 & 0.03 \\
\hline 0.36 & 0.36 & 0.13 & -0.36 & 1.07 & 0.98 & 0.33 \\
\hline-0.37 & 0.43 & 0.19 & -1.22 & 0.48 & -0.86 & 0.39 \\
\hline 0.48 & 0.12 & 0.01 & 0.24 & 0.72 & 3.97 & 0.00 \\
\hline
\end{tabular}

\section{Hedges's g and $95 \% \mathrm{Cl}$}

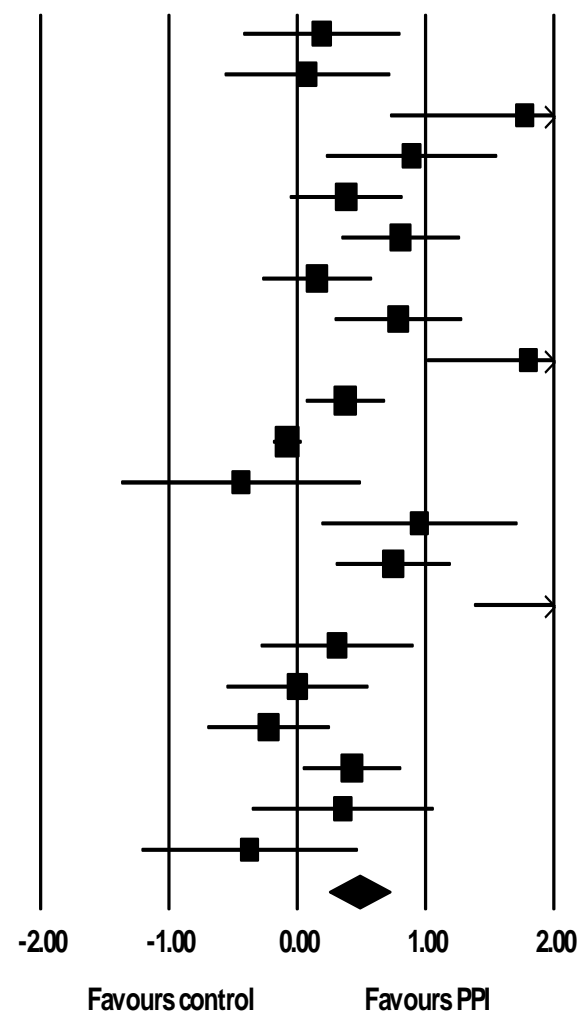

Figure 3. Effects of PPIs on psychological wellbeing, including outliers

Studyname

Statistics for each study

Hedges's g and $95 \% \mathrm{Cl}$

$\begin{array}{ccccc}\text { Hedges's } & \text { Standard } & \text { Lower } & \text { Upper } \\ g & \text { error } & \text { Variance limit limit Z-Value } p \text {-Value }\end{array}$

$\begin{array}{llllllll}\text { Arimitsu, 2016 } & 0.99 & 0.35 & 0.12 & 0.30 & 1.68 & 2.80 & 0.01 \\ \text { Asgharipoor, 2012 } & 0.11 & 0.45 & 0.20 & -0.78 & 0.99 & 0.23 & 0.81 \\ \text { Chiang, 2009 } & 1.50 & 0.23 & 0.05 & 1.04 & 1.96 & 6.39 & 0.00 \\ \text { Hendrik, 2017 } & 0.20 & 0.16 & 0.03 & -0.11 & 0.51 & 1.27 & 0.21 \\ \text { Hwang, 2016 } & -0.42 & 0.48 & 0.23 & -1.36 & 0.52 & -0.88 & 0.38 \\ \text { Im, 2016 } & 0.57 & 0.29 & 0.08 & 0.01 & 1.13 & 1.99 & 0.05 \\ \text { La, 2003 } & -0.04 & 0.25 & 0.06 & -0.54 & 0.46 & -0.15 & 0.88 \\ \text { Wong, 2016 } & 0.03 & 0.25 & 0.06 & -0.45 & 0.51 & 0.12 & 0.91 \\ & 0.40 & 0.22 & 0.05 & -0.03 & 0.83 & 1.81 & 0.07\end{array}$

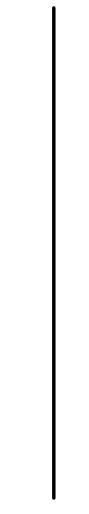

$-200$

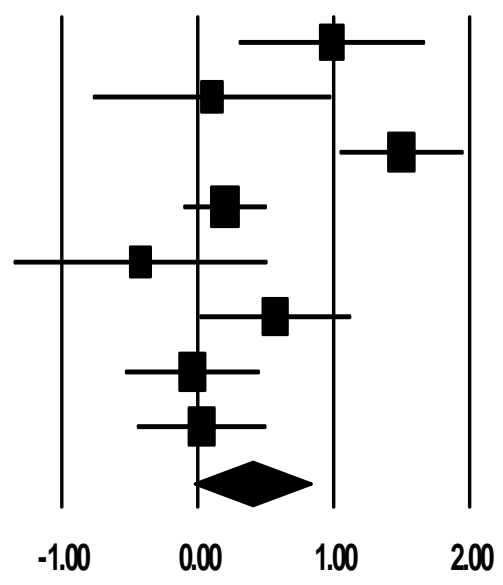

Favours control

Favours PPI 
Figure 4. Effects of PPIs on depression, including outliers

\begin{tabular}{|c|c|c|c|c|c|c|c|}
\hline \multirow[t]{2}{*}{ Studyname } & \multicolumn{7}{|c|}{ Statistics for each study } \\
\hline & $\begin{array}{l}\text { Hedges's } \\
\mathbf{g}\end{array}$ & $\begin{array}{l}\text { Standard } \\
\text { error }\end{array}$ & Variance & $\begin{array}{c}\text { Lower } \\
\text { limit }\end{array}$ & $\begin{array}{l}\text { Upper } \\
\text { limit }\end{array}$ & Z-Value & p-Value \\
\hline Arimitsu, २०16 & 0.78 & 0.34 & 0.12 & 0.11 & 1.46 & 2.27 & \\
\hline Asgharipoor, २०12 & -0.60 & 0.46 & 0.21 & -1.50 & 0.30 & -1.30 & \\
\hline Asl, 2014 & 0.90 & 0.34 & 0.12 & 0.23 & 1.57 & 2.62 & \\
\hline Cheng, 2015 & 0.86 & 0.25 & 0.06 & 0.36 & 1.35 & 3.41 & \\
\hline Chiang, 2009 & 1.05 & 0.22 & 0.05 & 0.62 & 1.49 & 4.76 & \\
\hline Choy, २०15 & 0.99 & 0.23 & 0.05 & 0.53 & 1.45 & 4.25 & \\
\hline Dowatabadi, 2016 & 1.10 & 0.37 & 0.13 & 0.38 & 1.81 & 3.00 & \\
\hline Guo, २०16 & 2.45 & 0.30 & 0.09 & 1.86 & 3.05 & 8.10 & \\
\hline Hendriks, 2017 & 0.37 & 0.16 & 0.03 & 0.06 & 0.69 & 2.35 & \\
\hline Khayatan, 2014 & 1.20 & 0.39 & 0.15 & 0.44 & 1.96 & 3.10 & \\
\hline Nikrahan, 2013 & -0.05 & 0.31 & 0.09 & -0.65 & 0.55 & -0.17 & \\
\hline Wong, 2016 & -0.39 & 0.22 & 0.05 & -0.83 & 0.05 & -1.72 & \\
\hline Zhang, 2014 & -0.89 & 0.45 & 0.20 & -1.77 & -0.01 & -1.97 & \\
\hline & 0.62 & 0.22 & 0.05 & 0.19 & 1.05 & 283 & \\
\hline
\end{tabular}

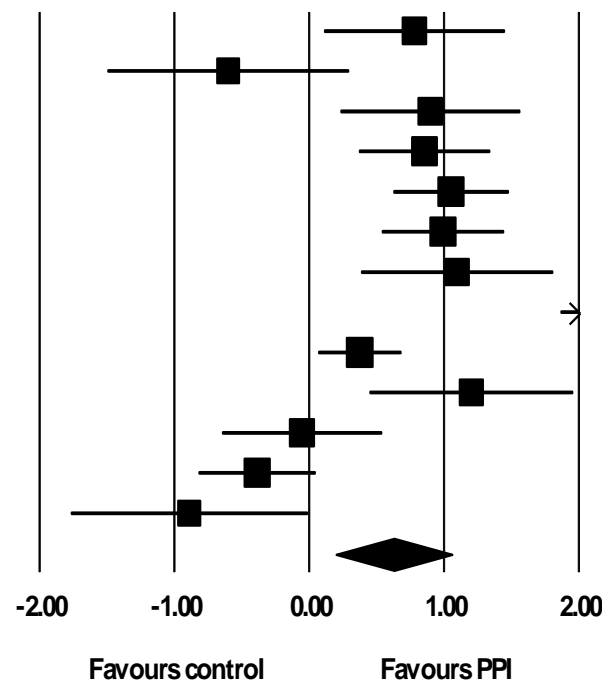

\subsubsection{Effects on anxiety}

Effects on anxiety were reported in five studies. A significant large effect was observed $(g=$ $0.93,95 \%$ CI: 0.28 to $1.59, p=0.006)$ at post-treatment. Effect sizes of studies ranged from 0.15 to 2.54 . Heterogeneity was significant and moderate $\left(\mathrm{I}^{2}=44.22, \mathrm{Q}=22.95, p<0.001\right)$. There were no outliers. The forest plot in Figure 5 displays the post-treatment effects.

Figure 5. Effects of PPIs on anxiety, including outliers

\begin{tabular}{|c|c|c|c|c|c|c|c|}
\hline \multirow[t]{2}{*}{ Study name } & \multirow[b]{2}{*}{$\begin{array}{c}\text { Hed ges's } \\
g\end{array}$} & \multicolumn{4}{|c|}{ Staisticsfor each study } & \multirow[b]{2}{*}{ Z-Value } & \multirow[b]{2}{*}{ p-Value } \\
\hline & & $\begin{array}{c}\text { Standard } \\
\text { error }\end{array}$ & Variance & $\begin{array}{c}\text { Lower } \\
\text { limit }\end{array}$ & $\begin{array}{c}\text { Upper } \\
\text { limit }\end{array}$ & & \\
\hline Arimitsu 2016 & 0.15 & 0.37 & 0.14 & -0.58 & 0.87 & 0.40 & 0.69 \\
\hline Hendils, 2017 & 0.33 & 0.16 & 0.03 & 0.02 & 0.65 & 210 & 0.04 \\
\hline Ji, 2016 & 1.79 & 0.44 & 0.19 & 0.93 & 266 & 4.06 & 0.00 \\
\hline Lau, 2011 & 0.58 & 0.26 & 0.07 & 0.06 & 1.09 & 218 & 0.03 \\
\hline Zhang, 2014 & 254 & 0.57 & 0.33 & 1.42 & 367 & 4.43 & 0.00 \\
\hline & 0.95 & 0.34 & 0.12 & 0.28 & 1.61 & 277 & 0.01 \\
\hline
\end{tabular}

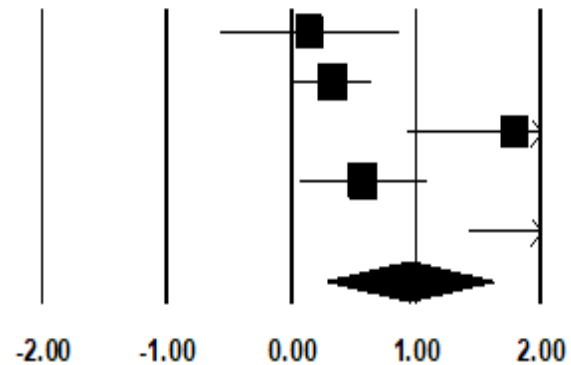

Favours control Favours PPI

\subsection{Subgroup analyses}

A moderator analysis was conducted for subjective wellbeing and depression, but not for psychological wellbeing, due to a limited amount of studies for this outcome. Also, due to a limited amount of studies from moderate $(n=3)$ and high quality $(n=2)$, the moderating effects of study quality were not reported. Our analysis showed that none of the variables had a moderating effect. All outcomes of the subgroup analyses are shown in Table 4 (below). 


\subsection{Publication bias}

For subjective wellbeing, the funnel plot was asymmetrically distributed in such a way that it was skewed in favor of studies with moderate to large effect sizes. This indicates the presence of publication bias. Egger's regression intercept also suggests that publication bias existed (intercept $=2.40, \mathrm{t}=3.72, \mathrm{df}=19, p=.0001$ ). The mean effect sizes were calculated by imputing missing studies using the Trim and Fill method. One study was imputed and the effect size was adjusted to $g=0.52$ (95\% CI: $0.28-0.75)$, meaning the effect size actually increased. Our findings are in line with other meta-analyses on the efficacy of wellbeing interventions (Bolier et al., 2013; Chakhssi et al., 2018; Weiss, Westerhof, \& Bohlmeijer, 2016), which consistently report more bias towards the publication of positive outcomes. Publication bias findings were not reported for psychological wellbeing, depression, and anxiety, due to the low numbers of studies (eight, 13, and five respectively). Such low numbers could lead to an unreliable publication bias analysis (Cuijpers, 2016; Lau, Ioannidis, Terrin, Schmid, \& Olkin, 2006).

Table 4. Results of moderator analysis

\begin{tabular}{|c|c|c|c|c|c|}
\hline Outcome & Criteria & Value & \# studies & Hedges' G $(95 \%$ CI $)$ & Z-Value (p-Value) \\
\hline \multirow{12}{*}{$\begin{array}{l}\text { Subjective } \\
\text { wellbeing }\end{array}$} & \multirow[t]{2}{*}{ Population } & Clinical & 6 & $0.88(0.45-1.31)$ & $3.99(0.00)^{* * *}$ \\
\hline & & Non-clinical & 15 & $0.32(0.07-0.58)$ & $2.50(0.01)^{*}$ \\
\hline & \multirow[t]{2}{*}{ Delivery } & Group & 16 & $0.64(0.37-0.92)$ & $4.65(0.00)^{* * *}$ \\
\hline & & Self-help & 5 & $-0.01(-0.18-0.16)$ & $-0.10(0.92) n s$ \\
\hline & \multirow[t]{2}{*}{ Intervention } & Single component & 11 & $0.33(0.10-0.56)$ & $2.84(0.00)^{* * *}$ \\
\hline & & Multi-component & 10 & $0.70(0.26-1.14)$ & $3.13(0.00)^{* * *}$ \\
\hline & \multirow[t]{2}{*}{ Control } & Active & 8 & $0.16(-0.20-0.51)$ & $0.87(0.38) \mathrm{ns}$ \\
\hline & & Non-active & 13 & $0.66(0.34-0.97)$ & $4.06(0.00)^{* * *}$ \\
\hline & \multirow[t]{2}{*}{ Duration } & $\leq 8$ weeks & 16 & $0.32(0.12-0.52)$ & $3.08(0.00)^{* *}$ \\
\hline & & $>8$ weeks & 5 & $1.27(0.24-2.30)$ & $2.41(0.02)^{* *}$ \\
\hline & \multirow[t]{2}{*}{ Adaptation } & Adapted & 10 & $0.30(-0.01-0.60)$ & $1.92(0.06)^{* *}$ \\
\hline & & Not adapted & 11 & $0.64(0.35-0.94)$ & $4.25(0.00)^{* * *}$ \\
\hline \multirow[t]{12}{*}{ Depression } & \multirow[t]{2}{*}{ Population } & Clinical & 6 & $0.63(0.10-1.16)$ & $2.34(0.02)^{*}$ \\
\hline & & Non-clinical & 7 & $0.62(-0.05-1.28)$ & $1.82(0.07)^{* *}$ \\
\hline & \multirow[t]{2}{*}{ Delivery } & Group & 11 & $0.70(0.23-1.16)$ & $2.93(0.00)^{* * *}$ \\
\hline & & Self-help & 2 & $0.23(-0.99-1.45)$ & $0.37(0.71) \mathrm{ns}$ \\
\hline & \multirow[t]{2}{*}{ Intervention } & Single component & 5 & $0.37(-0.32-1.07)$ & $1.06(0.29) \mathrm{ns}$ \\
\hline & & Multi-component & 8 & $0.78(0.18-1.38)$ & $2.52(0.01)^{*}$ \\
\hline & \multirow[t]{2}{*}{ Control } & Active & 4 & $-0.21(-1.02-0.61)$ & $-0.49(0.62) n s$ \\
\hline & & Non-active & 9 & $0.96(0.52-1.41)$ & $4.23(0.00)^{* * *}$ \\
\hline & \multirow[t]{2}{*}{ Duration } & $\leq 8$ weeks & 10 & $0.79(0.34-1.24)$ & $3.43(0.00) * * *$ \\
\hline & & $>8$ weeks & 3 & $0.04(-0.97-1.05)$ & $0.08(0.94) \mathrm{ns}$ \\
\hline & \multirow[t]{2}{*}{ Adaptation } & Adapted & 3 & $0.15(-0.59-0.89)$ & $0.41(0.67) \mathrm{ns}$ \\
\hline & & Not adapted & 10 & $0.76(0.23-1.29)$ & $2.81(0.00)^{* *}$ \\
\hline
\end{tabular}

\footnotetext{
${ }^{*} \mathrm{p}<0.05 ;{ }^{* *} \mathrm{p}<0.01 ;{ }^{* * *} \mathrm{p}<0.001 ;$ ns: non-significant.
} 


\subsection{Follow-up effects}

The follow-up effects of the PPIs were examined from four weeks after baseline/post-test up to three months after baseline/post-test (see Table 3). Follow-up effects for psychological wellbeing and anxiety could not be calculated due to the limited amount of studies, namely four and two respectively. For subjective wellbeing (eight comparisons) the effect size slightly decreased ( $g=0.43,95 \%$ CI: $0.08-0.77, p=0.01$ ). For depression (seven comparisons, after removal of one outlier), an increased effect size ( $g=0.77,95 \%$ CI: $0.23-1.30, p=0.005)$ was found. These findings suggest that PPI's in non-Western countries are also effective up to three months follow-up.

\section{Discussion}

This study aimed to examine the efficacy of PPIs from non-Western countries across randomized controlled trials. Following a systematic literature search, 28 RCTs were included in the meta-analysis. A moderate effect size was found for subjective wellbeing ( $g=$ $0.48)$ and psychological wellbeing $(g=0.40)$, and large effect sizes for depression $(g=0.62)$ and anxiety $(g=0.95)$. After removing outliers, the effect sizes decreased for subjective wellbeing $(g=0.36)$ and for psychological wellbeing $(g=0.22)$, but slightly increased for depression $(g=0.69)$. Follow-up results showed slightly decreased effect sizes for subjective wellbeing $(g=0.43)$ and an increased effect size for depression $(g=0.77)$. The overall study quality was low. Three studies were rated with a high quality, two with a moderate quality, and 23 with a low quality. There were also indications of publication bias, with a bias towards the publication of studies with positive results and with large effect sizes. Our findings on the larger effect sizes of studies from non-Western countries are in line with a previous metaanalysis on MPPIs (Hendriks et al., 2018b), that reported substantially larger effect sizes of PPIs from non-Western countries on subjective wellbeing, psychological wellbeing, and depression. The effects of PPIs on anxiety have not been reported in previous meta-analyses, most likely because of the low number of studies reporting this outcome. Since our metaanalysis was based on only five studies reporting on anxiety, caution is warranted when interpreting findings on anxiety in this study. The moderate to large effect sizes found in the current meta-analysis are also larger than those reported in a meta-analysis on PPIs by Bolier et al. (2013), which did not include non-Western studies. Hence, our findings indicate that the effect size of PPIs that are conducted in non-Western countries have larger effects than PPIs that are conducted in Western countries.

A possible explanation for the larger differences in effect sizes is the low quality of the studies from non-Western countries. The mean score of the study quality in this analysis was 1.79 , indicating that there is a high risk of bias in the studies and the overall study quality is rated as low. In comparison, a previous meta-analysis of MPPIs where study quality was determined using the same criteria revealed a mean score of 3.43 for quality of studies from Western countries (Hendriks et al, 2018b). A high risk of bias, or low study quality, is often associated with larger effect sizes, or vice versa. For example, a meta-analysis on the efficacy of psychotherapy (Cuijpers, van Straten, Bohlmeijer, Hollon, \& Andersson, 2010) reported relatively smaller effect sizes in studies of higher quality, compared with low-quality studies. A meta-analysis on the efficacy of PPIs (Bolier et al., 2013) suggested that the association between high effect sizes and lower study quality might also be applicable to PPIs. Our quality analysis showed that RCTs of PPIs from non-Western countries often do not adequately describe the sequence generation of randomization, or how allocation to the intervention was concealed. Inadequate random 
sequence and allocation concealment can overestimate treatment effects significantly (ArmijoOlivo et al., 2015; Dettori, 2010). Blinding of assessors was also not described in the large majority of the studies, which can lead to overestimation of treatment effects (Schulz \& Grimes, 2002). The found sample sizes were also small: 14 studies (50\%) had fewer than 20 participants in the intervention group, and 10 studies (36\%) had between 20 and 40 participants in the intervention group. The large majority of the studies in this meta-analysis was underpowered, which can lead to inflated estimates of the effect sizes (La Caze \& Duffull, 2011). Only five studies used intentionto-treat analysis (ITT), which is a statistical method where data from all randomized participants is analyzed, regardless of their adherence. Intention-to-treat analysis avoids an overestimation of the effects of an intervention (Gupta, 2011; Hollis \& Campbell, 1999).

In addition to the methodological biases, other biases may contribute to higher effect sizes in non-Western countries. A well-known phenomenon in psychology is the Hawthorne effect. The awareness of being observed leads to conformity and social desirability, which in turn leads to positive behavior outcomes (McCambridge, Witton, \& Elbourne, 2014). Research suggests that in collectivistic societies people tend to respond in more socially desirable ways, to maintain good relationships with others (Johnson \& Van de Vijver, 2003; Lalwani, Shavitt, \& Johnson, 2006). Another aspect of the Hawthorne effect is the novelty effect of an intervention. In studies on the effects of mobile health interventions in Western countries, it is noted that new technologies are perceived as more novel and having more value than traditional interventions. This novelty effect may lead to greater enthusiasm among participants and a greater attention to a particular intervention (Ammenwerth \& Rigby, 2016; Turner-McGrievy, Kalyanaraman, \& Campbell, 2013). In non-Western countries, this effect could also be applicable to regular psychological interventions, since access to mental health interventions is limited (de Jong et al., 2015; Rathod et al., 2017).

Despite the lower study quality and the influence of possible biases that may have contributed to overestimation of the effect sizes, the possibility that PPIs in non-Western countries have larger effect sizes than PPIs from Western countries simply because they are more effective cannot be excluded. We suggest that PPIs constitute a good cultural fit with nonWestern populations. Western European and North American cultures ("Western") are often described as independent, whereas Asian and South American cultures ("Eastern") are characterized as interdependent (Markus \& Kitayama, 1991; Morris \& Peng, 1994; Park, Uchida, \& Kitayama, 2016). While the goal of positive psychology interventions is to increase the wellbeing of the individual, many positive interventions operate through collective pathways that aim to improve interdependent relationships. The self, in interdependent cultures, is often perceived as a group-self with strong connections and feelings towards family members and the close environment. In such a setting, a group intervention may elicit more social support and wellbeing than in an individualized, independent, and egocentric cultural setting. Examples are positive psychology activities such as the gratitude visit (Davis et al., 2016; Emmons \& Stern, 2013), acts of kindness (Buchanan \& Bardi, 2010), and forgiveness (Derakhtkar \& Ahangarkani, 2016). In addition, many positive psychology activities aim to stimulate low arousal emotions such as kindness (Otake, Shimai, Tanaka-Matsumi, Otsui, \& Fredrickson, 2006; O'Connell, O'Shea, \& Gallagher, 2016), and compassion (Arimitsu, 2016; Yang, Liu, Shao, Ma, \& Tian, 2015), and integrate prayer and other spiritual activities (Rouholamini, Kalantarkousheh, \& Sharifi, 2017; Wu \& Koo, 2016) into interventions. Studies show people from Eastern cultures prefer such low arousal emotions (Lim, 2016; Tugade \& Fredrickson, 2004), and there is evidence suggesting cultural fit of emotions is associated with better health (Yoo \& Miyamoto, 2018). PPIs often include activities that aim to increase awareness, based on Buddhist philosophy, for example, 
mindfulness-based activities (Hamilton, Kitzman, \& Guyotte, 2006; Ivtzan \& Lomas, 2016), and loving kindness meditation (Fredrickson, Cohn, Coffey, Pek, \& Finkel, 2008). In addition, several studies from Iran were recently published that examined the effects of Islamic-based PPIs (AlSeheel \& Noor, 2016; Rouholamini et al., 2017; Saeedi, Nasab, Zadeh, \& Ebrahimi, 2015). Such intervention may constitute a cultural fit with the backgrounds of the participants. This, in turn, could result in greater enthusiasm, commitment, and participation among the study populations, and therefore contribute to higher effect sizes.

\subsection{Study limitations}

Besides the low quality of the studies, there are four additional limitations to the findings of this meta-analysis. First, our findings were based on a relatively small number of studies per outcome and subgroup. For example, psychological wellbeing was an outcome in only eight studies, depression in 11 studies, and anxiety in five studies. Sample sizes were relatively small in the exploratory subgroup analyses. This limits the interpretation of the differences between groups. Due to the small number of studies, publication bias analysis was not performed for psychological wellbeing, depression, and anxiety. Follow-up effects could only be calculated for subjective wellbeing and depression, and findings should be treated with caution in light of the limited numbers. For these reasons, definite conclusions on the effects of PPIs from non-Western countries cannot be drawn. Second, due to the high heterogeneity of the studies, it was not possible to clearly determine optimal conditions, for example, differences in efficacy between clinical or healthy populations, or differences in duration of the intervention. Third, only studies published in peer reviewed journals in the English language were included. Studies that were published in book chapters, dissertations, studies in grey literature and studies that were not in the English language (for example, two studies from Iran, which were only available in Arabic) were excluded. Fourthly, only RCTs were included in the analyses, and non-randomized controlled trials were excluded. While RCTs are considered the gold standard in clinical research (Rosen, Manor, Engelhard, \& Zucker, 2006), they are often cost intensive and complex (Korn \& Freidlin, 2012). Sufficiently powering an RCT with its concomitant costs may not always be feasible in low and middle income countries, due to lack of financial resources. For example, 625 articles on positive psychology in the Indian Journal of Positive Psychology were screened. These studies were conducted in India and other Asian countries. Only two of these studies were RCTs. Including quasi-experimental studies, which are perhaps more often conducted in non-Western countries than RCTs, could increase the number of studies in the subgroups and thereby provide a more complete overview of the efficacy of PPIs in non-Western countries.

\subsection{Recommendations}

The limited number of studies included contributed to the finding of no significant moderators and unreliable results for the publication bias analyses. Research in the field of positive psychology in non-Western countries is still in its infancy. A bibliometric analysis revealed that in the time period 1998 - 2013, only nine RCTs from non-Western countries were published, and that number has now (2018) almost quadrupled (Hendriks et al., 2018a). With this strong trend towards globalization of positive psychology, the study quality of non-Western country RCTs could benefit from protocol guidelines such as the Consolidated Standards of Reporting Trials (CONSORT) (Schulz, Altman, \& Moher, 2010) or the Standard Protocol Items: Recommendations for Interventional Trials (SPIRIT) guidelines (Chan et al., 2013). Further, we urge researchers from non-Western countries to publish in peer-reviewed journals, even when there is a null finding of no effect, as this is likely to reduce the publication bias in positive psychology research. 


\section{Authors}

Tom Hendriks

Anton de Kom University of Suriname

tom.hendriks@uvs.edu

Marijke Schotanus-Dijkstra

University of Twente

Aabidien Hassankhan

Anton de Kom University of Suriname

Tobi Graafsma

Anton de Kom University of Suriname

Ernst Bohlmeijer

University of Twente

Joop de Jong

University of Amsterdam

\section{Publishing Timeline}

Received 1 January 2018

Accepted 21 May 2018

Published 13 July 2018

\section{References}

Studies preceded by an asterisk were included in the systematic review and meta-analysis.

Adams, D. L. (1969). Analysis of a life satisfaction index. Journal of Gerontology, 24(4), 470-474. https://doi.org/10.1093/geronj/24.4.470

*Al-Seheel, A. Y., \& Noor, N. M. (2016). Effects of an Islamic-based gratitude strategy on Muslim students' level of happiness. Mental Health, Religion \& Culture, 19(7), 686-703. https://doi.org/10.1080/13674676.2016.1229287

Ammenwerth, E., \& Rigby, M. (2016). Evidence-based health informatics: Promoting safety and efficiency through scientific methods and ethical policy (Vol. 222). Amsterdam: Ios Press.

Andresen, E. M., Malmgren, J. A., Carter, W. B., \& Patrick, D. L. (1994). Screening for depression in well older adults: Evaluation of a short form of the CES-D (Center for Epidemiologic Studies Depression Scale). American Journal of Preventive Medicine, 10, 77-84. https://doi.org/10.1016/S0749-3797(18)30622$\underline{6}$

${ }^{*}$ Arimitsu, K. (2016). The effects of a program to enhance self-compassion in Japanese individuals: A randomized controlled pilot study. The Journal of Positive Psychology, 11(6), 559-571. https://doi.org/10.1080/17439760.2016.1152593

Armijo-Olivo, S., Saltaji, H., da Costa, B. R., Fuentes, J., Ha, C., \& Cummings, G. G. (2015). What is the influence of randomisation sequence generation and allocation concealment on treatment effects of physical therapy trials? A meta-epidemiological study. BMJ Open, 5(9), e008562. https://doi.org/10.1136/bmjopen-2015-008562

*Asgharipoor, N., Farid. A. A., Arshadi, H., \& Sahebi. A. (2012). A comparative study on the effectiveness of positive psychotherapy and group cognitive-behavioral therapy for the patients suffering from major depressive disorder. Iranian Journal of Psychiatry and Behavioral Sciences, 6(2), 33-41. 
*Asl, S. T. S., Sadeghi, K., Bakhtiari, M., Khazaie, H., Rezaei, M., \& Ahmadi, S.M. (2014). The effectiveness of group positive psychotherapy on improving the depression and increasing the happiness of the infertile women: Clinical trial. European Journal of Experimental Biology, 4(3), 269-275.

Beck, A. T., \& Steer, R. A. (1990). Manual for the Beck Anxiety Inventory. San Antonio, TX: Psychological Corporation.

Beck, A. T., Steer, R. A., \& Brown, G. K. (1996). Manual for the Beck Depression Inventory-II. San Antonio, TX: Psychological Corporation.

Block, J., \& Kremen, A. M. (1996). IQ and ego-resiliency: Conceptual and empirical connections and separateness. Journal of Personality and Social Psychology, 70(2), 349-361. https://doi.org/10.1037/0022$\underline{3514.70 .2 .349}$

Bolier, L., Haverman, M., Westerhof, G. J., Riper, H., Smit, F., \& Bohlmeijer, E. T. (2013). Positive psychology interventions: A meta-analysis of randomized controlled studies. BMC Public Health, 13, 119. https://doi.org/10.1186/1471-2458-13-119

Buchanan, K. E., \& Bardi, A. (2010). Acts of kindness and acts of novelty affect life satisfaction. Journal of Social Psychology, 150, 235-237. https://doi.org/10.1080/00224540903365554

Campbell, A., Converse, P. E., \& Rodgers, W. L. (1976). The quality of American life: Perceptions, evaluations, and satisfactions. New York, NY: Russell Sage Foundation.

Casellas-Grau, A., Font, A., \& Vives, J. (2014). Positive psychology interventions in breast cancer. A systematic review. Psychooncology, 23(1), 9-19. https://doi.org/10.1002/pon.3353

Chakhssi, F., Kraiss, J. T., Sommers-Spijkerman, M., \& Bohlmeijjer, E. T. (2018). The effect of positive psychology interventions on well-being in clinical populations: A systematic review and meta-analysis. Article under review.

Chan, A. W., Tetzlaff, J. M., Gøtzsche, P. C., Altman, D. G., Mann, H., Berlin, J. A., . . \& Krleža-Jerić, K. (2013). SPIRIT 2013 explanation and elaboration: Guidance for protocols of clinical trials. BMJ, 346, e7586. https://doi.org/10.1136/bmj.e7586

*Chan, D. (2013). Counting blessings versus misfortunes: Positive interventions and subjective well-being of Chinese school teachers in Hong Kong. Educational Psychology, 33(4), 504-519. https://doi.org/10.1080/01443410.2013.785046

${ }^{*}$ Cheng, S.-T., Tsui, P. K., \& Lam, J. H. (2015). Improving mental health in health care practitioners: Randomized controlled trial of a gratitude intervention. Journal of Consulting and Clinical Psychology, 83(1), 177-186. https://doi.org/10.1037/a0037895

${ }^{*}$ Chiang, K.-J., Chu, H., Chang, H.-J., Chung, M.-H., Chen, C.-H., Chiou, H.-Y., \& Chou, K.-R. (2010). The effects of reminiscence therapy on psychological well-being, depression, and loneliness among the institutionalized aged. International Journal of Geriatric Psychiatry, 25(4), 380-388. https://doi.org/10.1002/gps.2350

*Chiang, K. J., Lu, R. B., Chu, H., Chang, Y. C., \& Chou, K. R. (2008). Evaluation of the effect of a life review group program on self esteem and life satisfaction in the elderly. International Journal of Geriatric Psychiatry, 23(1), 7-10. https://doi.org/10.1002/gps.1824

${ }^{*}$ Choy, J. C., \& Lou, V. W. (2016). Effectiveness of the modified instrumental reminiscence intervention on psychological well-being among community-dwelling Chinese older adults: A randomized controlled trial. The American Journal of Geriatric Psychiatry, 24(1), 60-69. https://doi.org/10.1016/j.jagp.2015.05.008

Cuijpers, P. (2016). Meta-analyses in mental health research: A practical guide. Amsterdam, the Netherlands: Vrije Universiteit Amsterdam.

Cuijpers, P., van Straten, A., Bohlmeijer, E., Hollon, S., \& Andersson, G. (2010). The effects of psychotherapy for adult depression are overestimated: A meta-analysis of study quality and effect size. Psychological Medicine, 40(2), 211-223. https://doi.org/10.1017/S0033291709006114

Davis, D. E., Choe, E., Meyers, J., Wade, N., Varjas, K., Gifford, A., . . \& Worthington Jr, E. L. (2016). Thankful for the little things: A meta-analysis of gratitude interventions. Journal of Counseling Psychology, 63(1), 20-31. https://doi.org/10.1037/cou0000107 
Davis, J., Mengersen, K., Bennett, S., \& Mazerolle, L. (2014). Viewing systematic reviews and metaanalysis in social research through different lenses. SpringerPlus, 3(1), 511. https://doi.org/10.1186/2193-1801-3-511

de Jong, J. T., Berckmoes, L. H., Kohrt, B. A., Song, S. J., Tol, W. A., \& Reis, R. (2015). A public health approach to address the mental health burden of youth in situations of political violence and humanitarian emergencies. Current Psychiatry Reports, 17(7), 60. https://doi.org/10.1007/s11920-0150590-0

*Deng, Y.-H., Zhu, Y.-J., Li, Y., \& Liu, X.-P. (2016). Effects of gratitude-based intervention on aggression and subjective well-being in violent criminals. Chinese Journal of Clinical Psychology, 24(2), 368-372.

Derakhtkar, A., \& Ahangarkani, M. (2016). The effect of forgiveness training on the level of respect to spouse and marital satisfaction in women with marital problems. Iranian Journal of Psychiatry and Clinical Psychology, 22(1), 30-38.

Derogatis, L. R., \& Unger, R. (2010). Symptom Checklist-90-Revised. Corsini encyclopedia of psychology and behavioral science. https://doi.org/10.1002/9780470479216.corpsy0970

Dettori, J. (2010). The random allocation process: Two things you need to know. Evidence-based Spine-care Journal, 1(3), 7-9. https://doi.org/10.1055/s-0030-1267062

Dickens, L. R. (2017). Using gratitude to promote positive change: A series of meta-analyses investigating the effectiveness of gratitude interventions. Basic and Applied Social Psychology, 39(4), 193-208. https://doi.org/10.1080/01973533.2017.1323638

Diener, E., Emmons, R. A., Larsen, R. J., \& Griffin, S. (1985). The satisfaction with life scale. Journal of Personality Assessment, 49(1), 71-75. https://doi.org/10.1207/s15327752jpa4901 13

Diener, E., Wirtz, D., Tov, W., Kim-Prieto, C., Choi, D.-w., Oishi, S., \& Biswas-Diener, R. (2010). New well-being measures: Short scales to assess flourishing and positive and negative feelings. Social Indicators Research, 97(2), 143-156. https://doi.org/10.1007/s11205-009-9493-y

*Dowlatabadi, M. M., Ahmadi, S. M., Sorbi, M. H., Beiki, O., Razavi, T. K., \& Bidaki, R. (2016). The effectiveness of group positive psychotherapy on depression and happiness in breast cancer patients: A randomized controlled trial. Electronic Physician, 8(3), 2175-2180. https://doi.org/10.19082/2175

Duval, S., \& Tweedie, R. (2000). Trim and fill: A simple funnel plot-based method of testing and adjusting for publication bias in meta analysis. Biometrics, 56(2), 455-463. https://doi.org/10.1111/j.0006-341X.2000.00455.x

Egger, M., Smith, G. D., Schneider, M., \& Minder, C. (1997). Bias in meta-analysis detected by a simple, graphical test. BMJ, 315(7109), 629-634. https://doi.org/10.1136/bmj.315.7109.629

Emmons, R. A., \& Stern, R. (2013). Gratitude as a psychotherapeutic intervention. Journal of Clinical Psychology, 69(8), 846-855. https://doi.org/10.1002/jclp.22020

Enright, R. D., \& Rique, J. (2004). The Enright Forgiveness Inventory (EFI) User's Manual. Redwood City, CA: Mind Garden Press.

Fredrickson, B. L., Cohn, M. A., Coffey, K. A., Pek, J., \& Finkel, S. M. (2008). Open hearts build lives: Positive emotions, induced through loving-kindness meditation, build consequential personal resources. Journal of Personality and Social Psychology, 95(5), 1045-1062. https://doi.org/10.1037/a0013262

Gander, F., Proyer, R. T., Ruch, W., \& Wyss, T. (2013). Strength-based positive interventions: Further evidence for their potential in enhancing well-being and alleviating depression. Journal of Happiness Studies, 14(4), 1241-1259. https://doi.org/10.1007/s10902-012-9380-0

Ghosh, A., \& Deb, A. (2016). Positive psychology progress in India: Accomplishments and pathways ahead. Psychological Studies, 61(3), 113-125. https://doi.org/10.1007/s12646-016-0367-5

Gosling, S. D., Sandy, C. J., John, O. P., \& Potter, J. (2010). Wired but not WEIRD: The promise of the Internet in reaching more diverse samples. Behavioral and Brain Sciences, 33(2-3), 94-95. https://doi.org/10.1017/S0140525X10000300

*Guo, Y.-F., Zhang, X., Plummer, V., Lam, L., Cross, W., \& Zhang, J.-P. (2016). Positive psychotherapy for depression and self-efficacy in undergraduate nursing students: A randomized, controlled trial. International Journal of Mental Health Nursing, 26(4), 375-383. https://doi.org/10.1111/inm.12255 
Gupta, S. K. (2011). Intention-to-treat concept: A review. Perspectives in Clinical Research, 2(3), 109-112. https://doi.org/10.4103/2229-3485.83221

Hamilton, N. A., Kitzman, H., \& Guyotte, S. (2006). Enhancing health and emotion: Mindfulness as a missing link between cognitive therapy and positive psychology. Journal of Cognitive Psychotherapy, 20(2), 123-134. https://doi.org/10.1891/jcop.20.2.123

Hendriks, T., Schotanus-Dijkstra, M., Hassankhan, A., Graafsma, T., de Jong, J. , Bohlmeijer, E.T. (2018a). The efficacy of multicomponent positive psychology interventions: A meta-analysis. Manuscript submitted for publication.

${ }^{*}$ Hendriks, T., Schotanus-Dijkstra, M., Hassankhan, A., Sardjo, W., Graafsma, T., Bohlmeijer, E. T., \& de Jong, J. (2017). Psychological resilience and mental well-being in a multi-ethnic context: Findings from a randomized controlled trial. Manuscript submitted for publication.

Hendriks, T., Warren, M., Hassankhan, A., Schotanus-Dijkstra, M., Graafsma, T., Bohlmeijer, E. T., \& de Jong, J. (2018b). How WEIRD are positive psychology interventions? A bibliometric analysis. Manuscript accepted for publication.

Higgins, J., \& Thompson, S. G. (2002). Quantifying heterogeneity in a meta-analysis. Statistics in Medicine, 21(11), 1539-1558. https://doi.org/10.1002/sim.1186

Higgins, J. P. T., Altman, D. G., Gøtzsche P. C., Jüni, P., Moher, D., Oxman, A. D., Savovic, J., . . Sterne, J. A. C. (2011). The Cochrane Collaboration's tool for assessing risk of bias in randomized trials. BMJ, 343, d5928. https://doi.org/10.1136/bmj.d5928

Hills, P., \& Argyle, M. (2002). The Oxford Happiness Questionnaire: A compact scale for the measurement of psychological well-being. Personality and Individual Differences, 33(7), 1073-1082. https://doi.org/10.1016/S0191-8869(01)00213-6

*Ho, H. C. Y., Mui, M., Wan, A., Ng, Y.-L., Stewart, S. M., Yew, C., Hing Lam, T., \& Chan, S. S. (2016). Happy Family Kitchen II: A cluster randomized controlled trial of a community-based family intervention for enhancing family communication and well-being in Hong Kong. Frontiers in Psychology, 7, 638. https://doi.org/10.3389/fpsyg.2016.00638

Hollis, S., \& Campbell, F. (1999). What is meant by intention to treat analysis? Survey of published randomised controlled trials. BMJ, 319(7211), 670-674. https://doi.org/10.1136/bmj.319.7211.670

*Hwang, K., Kwon, A., \& Hong, C. (2016). A preliminary study of new positive psychology interventions: Neurofeedback-aided meditation therapy and modified positive psychotherapy. Current Psychology, 36(3), 683-695. https://doi.org/10.1007/s12144-016-9538-8

*Im, S. B., Cho, M.-K., Kim, S. Y., \& Heo, M. L. (2016). The Huddling Programme: Effects on empowerment, organisational commitment and ego-resilience in clinical nurses - a randomised trial. Journal of Clinical Nursing, 25(9-10), 1377-1387. https://doi.org/10.1111/jocn.13228

Ivtzan, I., \& Lomas, T. (2016). Mindfulness in positive psychology: The science of meditation and wellbeing. London, UK: Routledge.

*Ji, M., Hui, E., Fu, H., Watkins, D., Tao, L., \& Lo, S. K. (2016). Effects of a culture-adaptive forgiveness intervention for Chinese college students. British Journal of Guidance E Counselling, 44(3), 335-346. https://doi.org/10.1080/03069885.2015.1130798

Johnson, T. P., \& Van de Vijver, F. J. (2003). Social desirability in cross-cultural research. Cross-cultural Survey Methods, 325, 195-204.

Keyes, C. L. M. (2005). Mental illness and/or mental health? Investigating axioms of the complete state model of health. Journal of Consulting and Clinical Psychology, 73(3), 539-548. https://doi.org/10.1037/0022-006X.73.3.539

Keyes, C. L. M. (2007). Promoting and protecting mental health as flourishing: A complementary strategy for improving national mental health. American Psychologist, 62(2), 95-108. https://doi.org/10.1037/0003-066X.62.2.95

*Khayatan, T., Azkhosh, M., Bahmani, B., Azimian, M., \& Dolatshahi, B. (2014). Group positive psychotherapy and depression of females affected by multiple sclerosis. Iranian Rehabilitation Journal, 12(22), 49-53.

Kitwood, T., \& Bredin, K. (1997). Evaluating dementia care: The DCM method. Bradford, England: Bradford Dementia Research Group, Bradford University. 
Korn, E. L., \& Freidlin, B. (2012). Methodology for comparative effectiveness research: Potential and limitations. Journal of Clinical Oncology, 30(34), 4185-4187. https://doi.org/10.1200/ICO.2012.44.8233

*Koydemir, S., \& Sun-Selisik, Z. (2015). Well-being on campus: Testing the effectiveness of an online strengths-based intervention for first year college students. British Journal of Guidance \& Counselling, 44(4), 434-446. https://doi.org/10.1080/03069885.2015.1110562

La Caze, A., \& Duffull, S. (2011). Estimating risk from underpowered, but statistically significant, studies: Was APPROVe on TARGET? Journal of Clinical Pharmacy and Therapeutics, 36(6), 637-641. https://doi.org/10.1111/j.1365-2710.2010.01222.x

*Lai, C. K., Chi, I., \& Kayser-Jones, J. (2004). A randomized controlled trial of a specific reminiscence approach to promote the well-being of nursing home residents with dementia. International Psychogeriatrics, 16(1), 33-49. https://doi.org/10.1017/S1041610204000055

Lalwani, A. K., Shavitt, S., \& Johnson, T. (2006). What is the relation between cultural orientation and socially desirable responding? Journal of Personality and Social Psychology, 90(1), 165-178. https://doi.org/10.1037/0022-3514.90.1.165

Lau, J., Ioannidis, J. P., Terrin, N., Schmid, C. H., \& Olkin, I. (2006). Evidence based medicine: The case of the misleading funnel plot. BMJ, 333(7568), 597-600. https://doi.org/10.1136/bmj.333.7568.597

${ }^{*}$ Lau, R. W., \& Cheng, S.-T. (2011). Gratitude lessens death anxiety. European Journal of Ageing, 8(3), 169175. https://doi.org/10.1007/s10433-011-0195-3

Lee, H., Chiu, H., \& Kwong, P. (1994). Cross-validation of the Geriatric Depression Scale short form in the Hong Kong elderly. Bulletin Hong Kong Psychological Society, 32(33), 72-77.

Lim, N. (2016). Cultural differences in emotion: Differences in emotional arousal level between the East and the West. Integrative Medicine Research, 5(2), 105-109. https://doi.org/10.1016/j.imr.2016.03.004

Lipsey, M. W., \& Wilson, D. B. (1993). The efficacy of psychological, educational, and behavioral treatment: Confirmation from meta-analysis. American Psychologist, 48(12), 1181-1209. https://doi.org/10.1037/0003-066X.48.12.1181

Lovibond, P. F., \& Lovibond, S. H. (1995). The structure of negative emotional states: Comparison of the Depression Anxiety Stress Scales (DASS) with the Beck Depression and Anxiety Inventories. Behaviour Research and Therapy, 33(3), 335-343. https://doi.org/10.1016/0005-7967(94)00075-U

${ }^{*}$ Lü, W., Whang, Z., \& Liu, Y. (2013). A pilot study on changes of cardiac vagal tone in individuals with low trait positive affect: The effect of positive psychotherapy. International Journal of Psychophysiology, 88(2), 213-217. https://doi.org/10.1016/j.ijpsycho.2013.04.012

Lyubomirsky, S., \& Lepper, H. S. (1999). A measure of subjective happiness: Preliminary reliability and construct validation. Social Indicators Research, 46(2), 137-155. https://doi.org/10.1023/A:1006824100041

Macaskill, A. (2016). Review of positive psychology applications in clinical medical populations. Healthcare, 4(3), 66. https://doi.org/10.3390/healthcare4030066

Marín-Martínez, F., \& Sánchez-Meca, J. (1999). Averaging dependent effect sizes in meta-analysis: A cautionary note about procedures. The Spanish Journal of Psychology, 2, 32-38. https://doi.org/10.1017/S1138741600005436

Markus, H. R., \& Kitayama, S. (1991). Culture and the self: Implications for cognition, emotion, and motivation. Psychological Review, 98(2), 224-253. https://doi.org/10.1037/0033-295X.98.2.224

McCambridge, J., Witton, J., \& Elbourne, D. R. (2014). Systematic review of the Hawthorne effect: New concepts are needed to study research participation effects. Journal of Clinical Epidemiology, 67(3), 267277. https://doi.org/10.1016/j.jclinepi.2013.08.015

Moher, D., Hopewell, S., Schulz, K. F., Montori, V., Gøtzsche, P. C., Devereaux, P. J., ... Altman, D. (2010). CONSORT 2010 Explanation and Elaboration: Updated guidelines for reporting parallel group randomized trials. BMJ, 340, c869. https://doi.org/10.1136/bmj.c869

Moher, D., Liberati, A., Teztlaff, J., \& Altman, D. G. (2009). The PRISMA Group. Preferred reporting items for systematic reviews and meta-analyses: The PRISMA statement. Annals of Internal Medicine, 51, 1-7.

Molavi, H., Torkan, H., Soltani, I., \& Palahang, H. (2010). Structure standardization, validity and reliability of subjective well-being questionnaire. Iranian Journal of Psychiatry and Clinical Psychology, 16(3), 231-238. 
Morris, M. W., \& Peng, K. (1994). Culture and cause: American and Chinese attributions for social and physical events. Journal of Personality and Social Psychology, 67(6), 949-971. https://doi.org/10.1037/0022-3514.67.6.949

Neff, K. D. (2003). The development and validation of a scale to measure self-compassion. Self and Identity, 2(3), 223-250. https://doi.org/10.1080/15298860309027

*Nikrahan, G. R., Suarez, L., Asgari, K., Beach, S. R., Celano, C. M., Kalantari, M., ... Huffman, J. C. (2016). Positive psychology interventions for patients with heart disease: A preliminary randomized trial. Psychosomatics: Journal of Consultation and Liaison Psychiatry, 57(4), 348-358. https://doi.org/10.1016/j.psym.2016.03.003

O'Connell, B. H., O'Shea, D., \& Gallagher, S. (2016). Enhancing social relationships through positive psychology activities: A randomised controlled trial. The Journal of Positive Psychology, 11(2), 149-162. https://doi.org/10.1080/17439760.2015.1037860

Orwin, R. G. (1983). A fail-safe N for effect size in meta-analysis. Journal of Educational Statistics, 8(2), 157159. https://doi.org/10.2307/1164923

Otake, K., Shimai, S., Tanaka-Matsumi, J., Otsui, K., \& Fredrickson, B. L. (2006). Happy people become happier through kindness: A counting kindnesses intervention. Journal of Happiness Studies, 7(3), $361-$ 375. https://doi.org/10.1007/s10902-005-3650-z

*Otsuka, Y., Hori, M., \& Kawahito, J. (2012). Improving well-being with a gratitude exercise in Japanese workers: A randomized controlled trial. International Journal of Psychology and Counselling, 4(7), 86-91.

Park, J., Uchida, Y., \& Kitayama, S. (2016). Cultural variation in implicit independence: An extension of Kitayama et al. International Journal of Psychology, 51(4), 269-278. https://doi.org/10.1002/ijop.12157

Parks, A. C., \& Biswas-Diener, R. (2013). Positive interventions: Past, present and future. In T. B. Kashdan \& J. Ciarrochi (Eds.), The Context Press mindfulness and acceptance practica series. Mindfulness, acceptance, and positive psychology: The seven foundations of well-being (pp. 140-165). Oakland, CA: Context Press/New Harbinger Publications.

Rashid, T. (2009). Positive interventions in clinical practice. Journal of Clinical Psychology, 65(5), 461-466. https://doi.org/10.1002/jclp.20588

Rashid, T. (2015). Positive psychotherapy: A strength-based approach. The Journal of Positive Psychology, 10(1), 25-40. https://doi.org/10.1080/17439760.2014.920411

Rathod, S., Pinninti, N., Irfan, M., Gorczynski, P., Rathod, P., Gega, L., \& Naeem, F. (2017). Mental health service provision in low-and middle-income countries. Health Services Insights, 10. https://doi.org/10.1177/1178632917694350

Rosen, L., Manor, O., Engelhard, D., \& Zucker, D. (2006). In defense of the randomized controlled trial for health promotion research. American Journal of Public Health, 96(7), 1181-1186. https://doi.org/10.2105/AJPH.2004.061713

Rosenberg, M. S. (2005). The file-drawer problem revisited: A general weighted method for calculating fail-safe numbers in meta-analysis. Evolution, 59(2), 464-468. https://doi.org/10.1111/j.00143820.2005.tb01004.x

Rouholamini, M., Kalantarkousheh, S. M., \& Sharifi, E. (2017). Effectiveness of spiritual components training on life satisfaction of Persian orphan adolescents. Journal of Religion and Health, 56(6), 18951902. https://doi.org/10.1007/s10943-016-0256-1

Rusk, R. D., \& Waters, L. E. (2013). Tracing the size, reach, impact, and breadth of positive psychology. The Journal of Positive Psychology, 8(3), 207-221. https://doi.org/10.1080/17439760.2013.777766

Saeedi, H., Nasab, S.-H. M., Zadeh, A. M., \& Ebrahimi, H. A. (2015). The effectiveness of positive psychology interventions with Islamic approach on quality of life in females with multiple sclerosis. Biomedical and Pharmacology Journal, 8(2), 965-970. https://doi.org/10.13005/bpj/848

Schueller, S. M., Kashdan, T. B., \& Parks, A. C. (2014). Synthesizing positive psychological interventions: Suggestions for conducting and interpreting meta-analyses. International Journal of Wellbeing, 4(1), 9198. https://doi.org/10.5502/ijw.v4i1.5

Schueller, S. M., \& Parks, A. C. (2014). The science of self-help: Translating postive psychology research into increased individual happiness. European Psychologist, 19(2), 145-155.

https://doi.org/10.1027/1016-9040/a000181 
Schulz, K. F., Altman, D. G., \& Moher, D. (2010). CONSORT 2010 Statement: Updated guidelines for reporting parallel group randomized trials. Annals of Internal Medicine, 152(11), 726-732. https://doi.org/10.7326/0003-4819-152-11-201006010-00232

Schulz, K. F., \& Grimes, D. A. (2002). Blinding in randomised trials: Hiding who got what. The Lancet, 359(9307), 696-700. https://doi.org/10.1016/S0140-6736(02)07816-9

Shin, L. J., \& Lyubomirsky, S. (2017). Increasing well-being in independent and interdependent cultures. In M. A. Warren \& S. I. Donaldson (Eds.), Scientific advances in positive psychology (pp. 11-36). Santa Barbara, CA: Praeger.

Sin, N. L., \& Lyubomirsky, S. (2009). Enhancing well-being and alleviating depressive symptoms with positive psychology interventions: A practice-friendly meta-analysis. Journal of Clinical Psychology, 65(5), 467-487. https://doi.org/10.1002/jclp.20593

Spielberger, C. D. (2010). State Trait anxiety inventory. Hoboken, NJ: John Wiley \& Sons.

Sterne, J. A., Egger, M., \& Moher, D. (2008). Addressing reporting biases. In S. Green, J. P. Higgins, P. Alderson, M. Clarke, C. D. Mulrow, A. D. Oxman, ... \& J. Glanville. Cochrane handbook for systematic reviews of interventions: Cochrane book series (pp. 297-333). Chichester, England: John Wiley \& Sons Ltd.

Sutipan, P., Intarakamhang, U., \& Macaskill, A. (2017). The impact of positive psychological interventions on well-being in healthy elderly people. Journal of Happiness Studies, 18(1), 269-291. https://doi.org/10.1007/s10902-015-9711-z

Templer, D. I. (1970). The construction and validation of a death anxiety scale. The Journal of General Psychology, 82(2), 165-177. https://doi.org/10.1080/00221309.1970.9920634

Terasaki, M., Kishimoto, Y., \& Koga, A. (1992). Construction of a multiple mood scale. Shinrigaku kenkyu: The Japanese Journal of Psychology, 62(6), 350-356. https://doi.org/10.4992/jipsy.62.350

Thalheimer, W., \& Cook, S. (2002). How to calculate effect sizes from published research: A simplified methodology. bwgriffin.com/gsu/courses/edur9131/content/Effect Sizes pdf5.pdf

Tugade, M. M., \& Fredrickson, B. L. (2004). Resilient individuals use positive emotions to bounce back from negative emotional experiences. Journal of Personality and Social Psychology, 86(2), 320-333. https://doi.org/10.1037/0022-3514.86.2.320

Turner-McGrievy, G., Kalyanaraman, S., \& Campbell, M. K. (2013). Delivering health information via podcast or web: Media effects on psychosocial and physiological responses. Health Communication, 28(2), 101-109. https://doi.org/10.1080/10410236.2011.651709

Walsh, S., Cassidy, M., \& Priebe, S. (2016). The application of positive psychotherapy in mental health care: A systematic review. Journal of Clinical Psychology, 73(6), 638-651. https://doi.org/10.1002/jclp.22368

Watson, D., Clark, L. A., \& Tellegen, A. (1988). Development and validation of brief measures of positive and negative affect: The PANAS scales. Journal of Personality and Social Psychology, 54(6), 1063-1070. https://doi.org/10.1037/0022-3514.54.6.1063

Weiss, L. A., Westerhof, G. J., \& Bohlmeijer, E. T. (2016). Can we increase psychological well-being? The effects of interventions on psychological well-being: A meta-analysis of randomized controlled trials. PloS one, 11(6), e0158092. https://doi.org/10.1371/journal.pone.0158092

WHOQOL Group (1998). Development of the World Health Organization WHOQOL-BREF quality of life assessment. Psychological Medicine, 28(3), 551-558. https://doi.org/10.1017/S0033291798006667

*Wong, C. C., \& Mak, W. W. (2016). Writing can heal: Effects of self-compassion writing among Hong Kong Chinese college students. Asian American Journal of Psychology, 7(1), 74-82. https://doi.org/10.1037/aap0000041

Woodworth, R. J., O’Brien-Malone, A., Diamond, M. R., \& Schüz, B. (2016). Web-based positive psychology interventions: A Reexamination of effectiveness. Journal of Clinical Psychology, 73(3), 218232. https://doi.org/10.1002/jclp.22328

*Wu, L., \& Koo, M. (2016). Randomized controlled trial of a six-week spiritual reminiscence intervention on hope, life satisfaction, and spiritual well-being in elderly with mild and moderate dementia. International Journal of Geriatric Psychiatry, 31(2), 120-127. https://doi.org/10.1002/gps.4300 
Yang, L., Liu, Y.-y., Shao, J., Ma, M.-n., \& Tian, K. (2015). A pilot study on intervention effect of selfcompassion group training on depressed graduates. Chinese Journal of Clinical Psychology, 23(5), 947950.

Yoo, J., \& Miyamoto, Y. (2018). Cultural fit of emotions and health implications: A psychosocial resources model. Social and Personality Psychology Compass, 12(2), e12372. https://doi.org/10.1111/spc3.12372

*Yousefi, Z., Sharifi, K., Tagharrobi, Z., \& Akbari, H. (2015). The effect of narrative reminiscence on happiness of elderly women. Iran Red Crescent Medical Journal, 17(11), e19612. https://www.ncbi.nlm.nih.gov/pmc/articles/PMC4698128/

*Zhang, T., Fu, H., \& Wan, Y. (2014). The application of group forgiveness intervention for courtship-hurt college students: A Chinese perspective. International Journal of Group Psychotherapy, 64(3), 299-320. https://doi.org/10.1521/ijgp.2014.64.3.298 


\section{Appendix A}

\section{Search strategy}

Pubmed ((well-being[Title/Abstract] OR happiness[Title/Abstract] OR happy[Title/Abstract] OR flourishing[Title/Abstract] OR "life satisfaction"[Title/Abstract] OR "satisfaction with life" [Title/Abstract] OR optimism[Title/Abstract] OR gratitude[Title/Abstract] OR strengths[Title/Abstract] OR forgiveness[Title/Abstract] OR compassion[Title/Abstract] OR "positive psych" [Title/Abstract])) AND "random"*[Title/Abstract]

PsycINFO well-being or happiness or happy or flourishing or "life satisfaction" or "satisfaction with life" or optimism or gratitude or strengths or forgiveness or compassion or "positive psych").ti. and ("well-being" or happiness or happy or flourishing or "life satisfaction" or "satisfaction with life" or optimism or gratitude or strengths or forgiveness or compassion or "positive psych" ${ }^{*}$.ab. and random*af

Scopus \#1 well-being or happiness or happy or flourishing or "life satisfaction" or "satisfaction with life" or optimism or gratitude or strengths or forgiveness or compassion or "positive psych*" \#2 AND ABS(well-being or happiness or happy or flourishing or "life satisfaction" or "satisfaction with life" or optimism or gratitude or strengths or forgiveness or compassion or "positive psych*")AND TITLE-ABS-KEY(random*)) AND DOCTYPE(ar) AND PUBYEAR > 1997 AND ( LIMIT-TO ( SUBJAREA, "MEDI" ) OR LIMIT-TO ( SUBJAREA,"HEAL" ) OR LIMIT-TO ( SUBJAREA, "PSYC" ) OR LIMIT-TO ( SUBJAREA, "SOCI") OR LIMIT-TO ( SUBJAREA,"NURS" ) OR LIMIT-TO ( SUBJAREA,"BUSI" ) OR LIMIT-TO ( SUBJAREA,"MULT" ) ) AND ( LIMIT-TO ( LANGUAGE,"English" ) ) AND ( LIMIT-TO ( AFFILCOUNTRY,"United States" ) ) AND ( LIMIT-TO ( EXACTKEYWORD,"Human" ) OR LIMIT-TO ( EXACTKEYWORD, "Article" ) OR LIMIT-TO ( EXACTKEYWORD,"Humans" ) OR LIMIT-TO ( EXACTKEYWORD, "Controlled Study" ) OR LIMIT-TO ( EXACTKEYWORD,"Male" ) OR LIMIT-TO ( EXACTKEYWORD,"Female" ) OR LIMIT-TO ( EXACTKEYWORD,"Adult" ) OR LIMIT-TO ( EXACTKEYWORD,"Randomized Controlled Trial" ) OR LIMIT-TO ( EXACTKEYWORD,"Controlled Clinical Trial" ) OR LIMIT-TO ( EXACTKEYWORD, "Middle Aged" ) OR LIMIT-TO ( EXACTKEYWORD, "Aged" ) OR LIMITTO ( EXACTKEYWORD, "Clinical Trial") OR LIMIT-TO ( EXACTKEYWORD,"Physiology" ) OR LIMIT-TO ( EXACTKEYWORD,"Priority Journal" ) OR LIMIT-TO ( EXACTKEYWORD,"Major Clinical Study" ) OR LIMIT-TO ( EXACTKEYWORD,"Young Adult" ) OR LIMIT-TO ( EXACTKEYWORD,"Treatment Outcome" ) OR LIMIT-TO ( EXACTKEYWORD, "Methodology" ) OR LIMIT-TO ( EXACTKEYWORD, "Quality Of Life" ) OR LIMIT-TO ( EXACTKEYWORD,"Clinical Article" ) OR LIMIT-TO ( EXACTKEYWORD, "Procedures" ) OR LIMIT-TO ( EXACTKEYWORD,"Questionnaire" ) OR LIMIT-TO ( EXACTKEYWORD, "Human Experiment" ) OR LIMIT-TO( EXACTKEYWORD, "Normal Human" ) OR LIMIT-TO ( EXACTKEYWORD, "Wellbeing" ) OR LIMIT-TO ( EXACTKEYWORD, "Double Blind Procedure" ) OR LIMIT-TO ( EXACTKEYWORD,"Randomization" ) OR LIMIT-TO ( EXACTKEYWORD,"Depression" ) OR LIMIT-TO ( EXACTKEYWORD,"Outcome Assessment" ) OR LIMIT-TO ( EXACTKEYWORD, "Random Allocation" ) OR LIMIT-TO ( EXACTKEYWORD, "Follow Up" ) OR LIMIT-TO ( EXACTKEYWORD, "Questionnaires" ) OR LIMIT-TO ( EXACTKEYWORD, "Exercise Therapy" ) OR LIMIT-TO ( EXACTKEYWORD,"Time" ) OR LIMIT-TO ( EXACTKEYWORD, "Animals" ) OR LIMIT-TO ( EXACTKEYWORD,"DoubleBlind Method" ) OR LIMIT-TO ( EXACTKEYWORD,"Well-being" ) OR LIMIT-TO ( EXACTKEYWORD,"Psychology") OR LIMIT-TO ( EXACTKEYWORD,"Psychological Aspect" ) OR LIMIT-TO ( EXACTKEYWORD,"Stress, Mechanical" ) OR LIMIT-TO ( EXACTKEYWORD, "Training" ) OR LIMIT-TO ( EXACTKEYWORD,"Physical Activity" ) OR LIMIT-TO ( EXACTKEYWORD, "Strength" ) OR LIMIT-TO ( EXACTKEYWORD, "Mental Health" ) OR LIMIT-TO ( EXACTKEYWORD, "Placebo" ) OR LIMIT-TO ( 
EXACTKEYWORD, "Health Status" ) OR LIMIT-TO ( EXACTKEYWORD,"Happiness" ) OR LIMIT-TO ( EXACTKEYWORD,"Personal Satisfaction" ) OR LIMIT-TO (

EXACTKEYWORD,"Self Concept" ) OR LIMIT-TO ( EXACTKEYWORD,"Life Satisfaction" )

OR LIMIT-TO ( EXACTKEYWORD, "Follow-Up Studies" ) OR LIMIT-TO (

EXACTKEYWORD,"Anxiety" ) OR LIMIT-TO ( EXACTKEYWORD,"Satisfaction" ) OR

LIMIT-TO ( EXACTKEYWORD, "Psychological Well Being" ) OR LIMIT-TO (

EXACTKEYWORD, "Self Report" ) OR LIMIT-TO ( EXACTKEYWORD,"Instrumentation" )

OR LIMIT-TO ( EXACTKEYWORD,"Emotion" ) OR LIMIT-TO (

EXACTKEYWORD," Adaptation, Psychological" ) OR LIMIT-TO ( EXACTKEYWORD, "United

States" ) OR LIMIT-TO ( EXACTKEYWORD,"Fatigue" ) OR LIMIT-TO (

EXACTKEYWORD,"Social Support" ) OR LIMIT-TO ( EXACTKEYWORD, "Affect" ) OR

LIMIT-TO ( EXACTKEYWORD, "Pilot Study" ) 